\title{
Best Wavelet Packet Bases in a Rate-Distortion Sense
}

\author{
Kannan Ramchandran and Martin Vetterli, Senior Member, IEEE
}

\begin{abstract}
The use of an adaptive tree structure using wavelet packets as a generalized wavelet decomposition for signal compression was recently introduced by Coifman, Meyer, Quake, and Wickerhauser [1]. The idea is to decompose a discrete signal using all possible wavelet packet bases of a given wavelet kernel, and then to find the "best" wavelet packet basis. Unlike the work in [1], in this paper we employ a framework that includes both rate and distortion. A fast algorithm is formulated to "prune" the complete tree, signifying the entire library of admissible wavelet packet bases, into that best basis subtree which minimizes the global distortion for a given coding bit budget or conversely which minimizes the total coding bit rate for a target quality. Arbitrary finite quantizer sets are assumed to each hierarchical level of the basis-family tree. Finally, image coding applications using wavelet packets as well as a DCT basis family quadtree segmentation in a JPEG-like environment are described with favorable results.
\end{abstract}

\section{INTRODUCTION}

S OURCE coding for stripping redundancy from typical highly-correlated sources like speech and image waveforms has been studied extensively. Some popular techniques addressed in the literature include vector quantization (VQ), linear predictive coding, linear transform coding (like the KLT and the DCT), and subband coding as well as various hybrid combinations of these.

$\mathrm{VQ}$ is a popular and powerful scheme for compressing correlated discrete signal sets whose characteristics have been "trained" initially, but its complexity grows exponentially with vector dimensionality. Linear transformations like the DCT are less computationally demanding, but owing to their "fixed" nonadaptive nature, their compression potential relies heavily on the stationarity of the signal. For nonstationary sources, linear transforms or prediction techniques generally fail to exploit all of the source redundancy present. If one could combine the adaptability of VQ with the speed of linear transform coding, one could achieve a coding scheme which adapts to signal nonstationarities without sacrificing computational ease. Wavelet packets, introduced by Coifman, Meyer, Quake, and Wickerhauser (CMQW) [1], [2] to be described in Section II, permit this combination, and offer a flexible yet computationally nonoverwhelming framework in which to undertake efficient signal compression.

This paper is organized as follows: Section II provides a brief description of the background information on which the

Manuscript received January 23, 1992; revised October 23, 1992. This work was supported in part by New York Science and Technology Foundation's CAT and in part by the National Science Foundation under Grant ECD-8811111 and under Grant MIP-90-14189. The associate editor coordinating the review of this paper and approving it for publication was Dr. Michael Unser.

The authors are with the Department of Electrical Engineering and Center for Telecommunications Research, Columbia University, New York, NY $10027-6699$

IEEE Log Number 9206907. rest of the paper is founded, while outlining the scope of applicability of this work. Section III highlights the intuition and main idea of the algorithm. Section IV states the problem formally, while Section $V$ undertakes a fast solution to the problem. Section VI details the flowchart of the complete algorithm. Finally, Section VII provides image coding applications using wavelet packets as well as DCT-based quadtree segmentation, based on our fast algorithm.

\section{BACKGROUND AND SCOPE OF THIS WORK}

This section deals with a brief explanation of wavelet packets, a summary of bit allocation techniques based on operational rate-distortion theory, a brief look at the related existing literature and the contribution and scope of applicability of this paper.

\section{A. Wavelet Packets}

Wavelet packets (WP) were introduced recently by Coifman, Meyer, Quaker, and Wickerhauser (CMQW) [1], [2] as a family of orthonormal (ON) bases for discrete functions of $R^{N}$, and include the well-known wavelet basis and the ShortTime-Fourier-Transform (STFT)-like basis ${ }^{1}$ as its members. While a brief description of wavelet packets, together with an intuitive feel for what they represent, will be provided here, the interested reader is referred to [1] and [2] for a detailed mathematical treatment of the subject.

Wavelet packets represent a generalization of the method of multiresolution decomposition, and comprise the entire family of subband coded (tree) decompositions. They offer fast access to a rich menu of ON bases, from which the "best basis" can be chosen. If one represents the complete subband decomposition of a discrete signal set in $R^{N}$ as a regular analysis tree of depth $\log N$, the CMQW approach permits the choice of a decomposition topology corresponding to any pruned subtree of the original tree, i.e., any subtree sharing the same root as the original tree. This is obviously isomorphic to all permissible subband topologies (see Fig. 1), with the collection of terminal or leaf nodes of every pruned subtree representing the entire library of permissible $\mathrm{ON}$ bases.

Thus this decomposition might be used to code independent segments of a given nonstationary signal. It enables the coder to exhibit, for example, a STFT-like characteristic (regular tree) at one source instance, a wavelet characteristic (logarithmic tree) at another instance, or any intermediate characteristic (arbitrary WP subtree) at yet other instances, to best match the signal's nonstationary statistics. See Fig. 1. The ON property is vital to the development of the fast

\footnotetext{
'That is, the basis which results in uniform frequency resolution.
} 


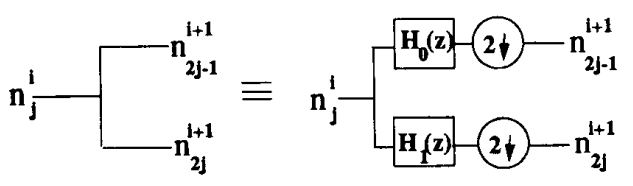

(a)

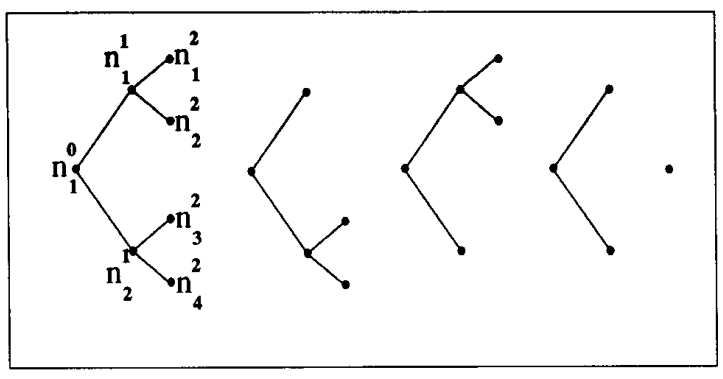

(b)

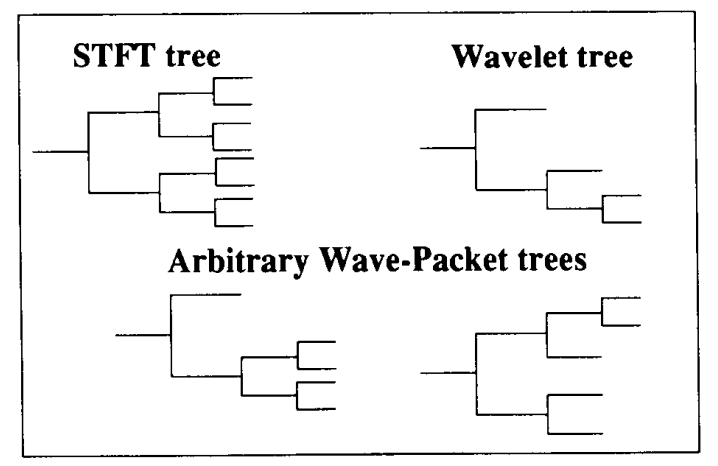

(c)

Fig. 1. (a) Tree notation for analysis filter bank: The lower branch, $H_{0}$ is the low-pass filter, and the upper branch, $H_{1}$ is the high-pass filter. (b) All possible binary wavelet packet decompositions of depth 2 . Note that $n_{j}^{\prime}$ refers to the $j$ th node at the $i$ th scale of the tree, as shown in the full tree. (c) Some permissible depth-3 decompositions.

pruning algorithm, as it enables the coding rate and distortion associated with any tree to be additive over the rates and distortions of the leaf nodes, with respect to an $l_{2}$ norm (like mean-squared error (MSE) or weighted MSE) criterion.

\section{B. Quantization and Bit Allocation}

The problem of bit allocation, where a given bit budget must be distributed efficiently among a set of given admissible quantization choices, is a classical problem in signal compression that has received exhaustive treatment in source coding literature [3]-[5]. A classical framework for source coding is Shannon's rate-distortion theory [6], which deals with minimization of source distortion subject to a channel rate constraint, or the dual problem of minimization of channel rate subject to a distortion constraint. A practical coding environment involves a finite set of admissible quantizers, characterized by their (operational) rate-distortion functions [7], ranging from convex [3] to completely arbitrary [4]. These quantizers are used by the allocation algorithm to determine the best strategy to minimize the overall coding distortion subject to a total bit rate budget constraint. We use this framework to seek our best basis WP and best quantizer choices.

\section{Related Work and Contribution of this Paper}

While the adaptivity and speed of the best-basis search of [1] are unmistakable, the cost criterion and the coding (quantization) method used there to exploit this speed and flexibility are somewhat ad hoc. In [1], one choice of the best basis is the one which results in the fewest number of nonzero coefficients after thresholding is performed with respect to a desired threshold. Other choices include the use of onesided metrics like entropy only or MSE only. None of the above schemes is optimal in a rate-distortion sense, nor do they address the problem for arbitrary sets of quantization choices, as is done here. In our framework, the best basis and its optimal quantization choice are found jointly using an $\mathrm{R}-\mathrm{D}$ criterion of smallest distortion for a given bit budget or vice versa. While our algorithm is inspired by a search for the best basis wavelet packet, it obviously applies to quadtree segmentations as well. In fact, our approach could be viewed, in its quadtree application, as an extension of the work by Shoham and Gersho [4] to proved a fast algorithm covering hierarchies of admissible quantizers. It may also be regarded, in this context, as a generalization of Chou et al.'s (G-BFOS) algorithm [5], [8] to the case where monotonicity constraints of rate and distortion with tree depth are removed. Fig. 2(a) gives an example of a rate-distortion characteristic that is constrained to be monotonic with tree depth, ${ }^{2}$ as identified by a single transition form the "merge" to "split" boundaries, a constraint that is necessary for the quadtree algorithm mentioned in [5]. Fig. 2(b) shows a nonrestrictive case, where arbitrary transitions between the "split" and "merge" regions are permitted.

While bit allocation strategies for various coding environments have been formulated in the literature, the problem of using arbitrary quantizers in a generalized multiresolution wavelet decomposition framework in an $\mathrm{R}-\mathrm{D}$ optimal way has not, to the best of the author's knowledge, been addressed. While the treatment uses combinations of well known methods, the current interest in WP's is such that we believe our contribution to be useful. Thus the merit of this work could be viewed in its merging of the concept of orthonormal tiling of the time-frequency plane using wavelet packets, a recent result from the "wavelet" community [2] with the discipline of R-D optimal bit allocation, the bread and butter of the "quantization" community [7].

A practical contribution of this paper involves the description of the results of WP-based image compression using the Daubechies D4 set of wavelet filters. We also perform quadtree-based image compression using a family of DCT bases (as in [9], which is not, however, R-D optimal). This application is similar to that of work independently done by Sullivan and Baker [10], who performed efficient quadtree segmentation using VQ. Our example uses classified

\footnotetext{
2 That is, as the tree grows, the rate increases and the distortion decreases.
} 


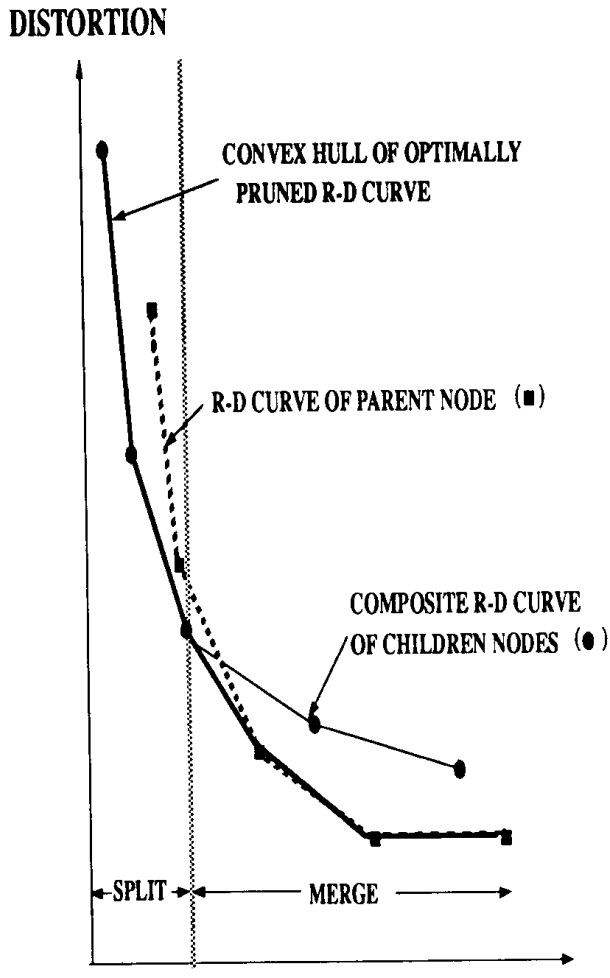

RATE

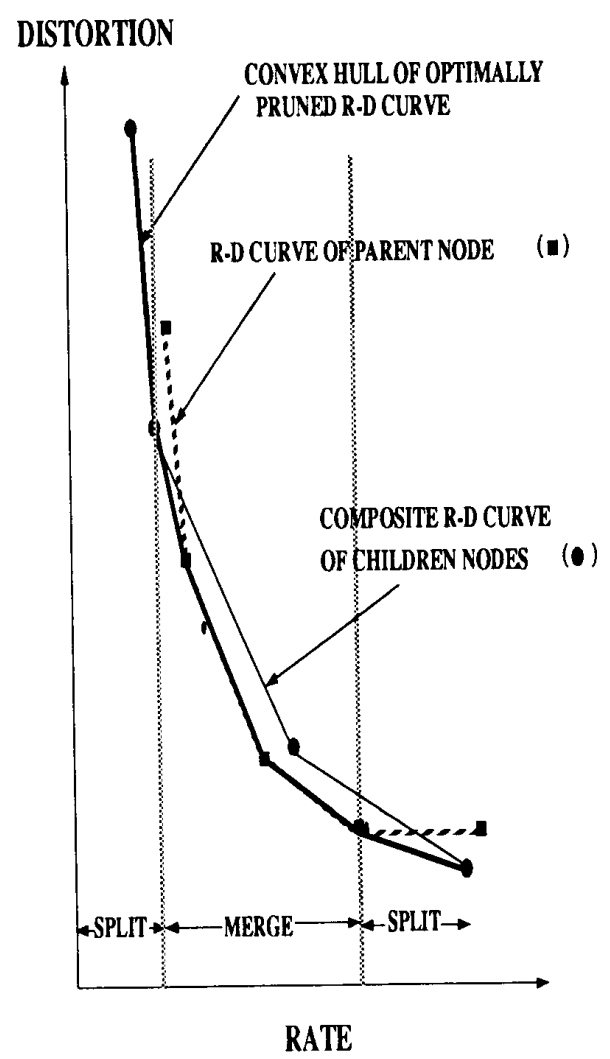

(b)

Fig. 2. "Split/Merge" boundaries shown for (a) monotonic case to which the G-BFOS algorithm is constrained, and (b) nonrestrictive case.

quantizers in a hierarchical JPEG (DCT-based) [11] coding environment.

It must be emphasized that the standard basis tree employed by [10] is not a WP tree, which is derived recursively using Quadrature Mirror Filters (QMF) filter banks or using multiresolution wavelet analysis (whose equivalence has been established [12], [13]). The scope of applicability of our algorithm extends to all classes of structures which permit the construction of a hierarchy of basis covers for the input signal space. While this obviously includes structures like quadtrees and orthonormally transformed (e.g., DCT) quadtrees, other powerful structures such as the CMQW multiresolution decomposition wavelet packets and hierarchical subband coders are also applicable. In fact, the optimal tree pruning and bit allocation results of this paper apply to the entire family of hierarchical lapped transforms (HLT's) [14], [15]. As an example, our algorithm could be used to determine quantitatively, such important coder design considerations as the optimal decomposition depth for subband coding, or a performance comparison of filter banks of different kernels and topologies, or to determine an efficient DCT quadtree structure in a "hierarchical" JPEG application. Also, it can be extended to the "double tree" algorithm [16] to find optimal orthonormal signal splits, a topic of considerable interest in time-frequency analysis as well as signal compression.

\section{BASIC IDEA OF THE ALgORITHM}

We consider a rate-distortion framework with independent coding of the nodes of the wavelet packet tree and an MSE distortion metric. Due to additivity of the rate and distortion measures over disjoint covers of the input, the "hard" constrained problem of seeking the best WP basis which minimizes the average distortion $D$ for a target average bit rate $R$ (or vice versa) can be converted to an "easy" equivalent unconstrained problem by "merging" rate and distortion through the Lagrange multiplier $\lambda[4]$. Thus the unconstrained problem becomes the minimization of the Lagrangian cost function defined as

$$
J(\lambda)=D+\lambda R
$$

It can be shown that at R-D optimality, all signal elements (or leaves of the wavelet packet tree) must operate at a constant slope point $\lambda$ on their R-D curves (see Fig. 3). For a given $\lambda=|\Delta D / \Delta R|$, we populate each node of each tree block independently with the Lagrangian cost function associated with the best quantizer for that node. The best quantizer for a particular tree node is that one which "lives" at absolute slope $\lambda$ on the convex hull of the operational R-D curve for that node, as shown in Fig. 3. Then, by applying the pruning 


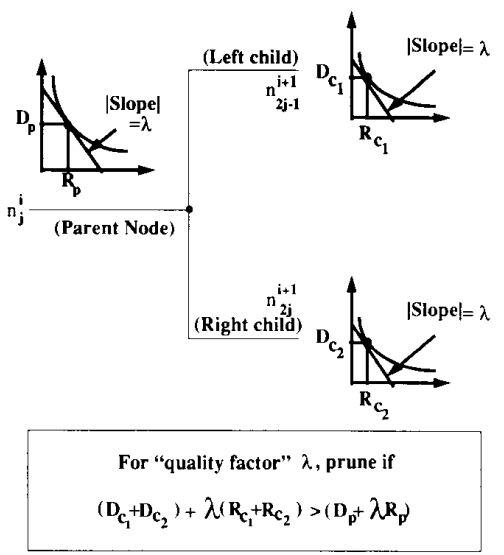

Fig. 3. Lagrangian cost pruning criterion for "quality criterion" $\lambda$ for each parent node of the wavelet packet tree. This condition is used recursively to do fast pruning from the complete tree depth towards the root to find the optimal subtree for a given $\lambda$.

criterion of Fig. 3 recursively on every node, starting from the full-depth tree and proceeding towards the root, we find the sequence of best wavelet packet bases and associated best quantizers with which to code the signal. The desired optimal constant slope value $\lambda^{*}$ is not known a priori and depends on the desired target budget or quality constraint. Fortunately, however, $\lambda^{*}$ can be obtained relatively painlessly via a fast convex recursion in $\lambda$ using the bisection algorithm, as will be shown later.

\section{Formal Problem Definition}

Without loss of generality, we will consider the problem of a binary wavelet packet decomposition tree of a 1-D discrete input signal (vector) of size $N$ in $l^{2}(N)\left\langle s_{1}, s_{2}, \cdots, s_{N}\right\rangle$. Though omitted for convenience, each branch of the analysis tree consists of the appropriate filter: high-pass filter (HPF) $H_{0}$ for the upper child and low-pass filter (LPF) $H_{1}$ for the lower child, followed by a decimator by 2 (see Fig. 1), with the corresponding synthesis tree consisting of an upsampler followed by the corresponding synthesis filters.

The analysis and synthesis filters of each branch satisfy the standard orthonormality conditions of paraunitary perfect reconstruction filter banks (PRFB's [13]). As is well known, iterating the orthonormal filter templates to the complete tree depth results in an equivalent generalized multiresolution decomposition tree (i.e., wavelet packet tree) whose nodes represent a family of orthonormal bases [1], [2]. We assume that there are $M$ signal blocks to be coded independently, each of size $N$. As the algorithm works in parallel for all independent blocks, it will suffice to analyze a single block. To help provide a clear notation-free understanding of our algorithm, we introduce a "toy" example that we will invoke at various points in this paper, which uses a length- 4 signal block with maximum tree depth of 2 (see Fig. 4). The example uses the popular Haar basis $(\{[1 / \sqrt{(2)}, 1 / \sqrt{(2)}],[1 / \sqrt{(2)},-1 / \sqrt{(2)}]\})$ or sum and difference filters as the wavelet kernel. See Fig. 5 for the five possible depth-2 Haar WP bases.

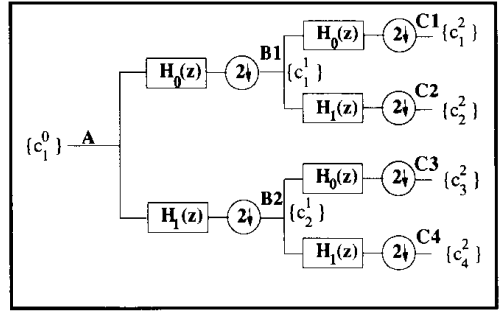

(a)

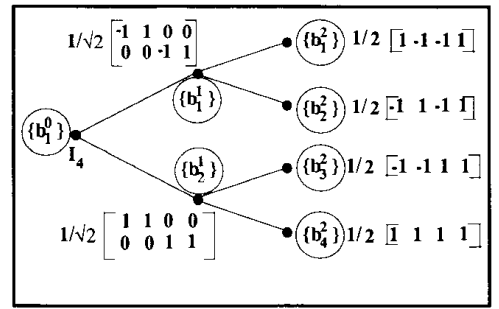

(b)

Fig. 4. Toy example showing a depth-2 wavelet packet decomposition for a length-4 signal block using the popular Haar wavelet basis $\left(H_{1}(z)=1 / \operatorname{sqrt}(2)\left(1+z^{-1}\right)\right.$ and $\left.H_{0}(z)=1 / \operatorname{sqrt}(2)\left(1-z^{-1}\right)\right)$. (a) Full tree showing the root A, level-one B, and level-two C. (b) Orthonormal wavelet packet basis vectors associated with each tree node. Note that the $\left\{c_{j}^{i}\right\}$ 's represent the inner product of the signal with respect to the basis vectors $\left\{b_{j}^{i}\right\}$.

Let us define the following terms to be used in the formulation:

- $T$ : Complete WP tree (STFT tree) for each signal block of depth $\log N$.

- $S \preceq T$ : Pruned subtree of $T$, i.e., any admissible WP bases subtree.

- $\tilde{S}$ : Set of leaves or terminal nodes of subtree $S$.

- $n_{j}^{i}, b_{j}^{i}, c_{j}^{i}$ : The $j$ th (of possible $2^{i}$ choices) node, basis, and coefficient vector respectively, at the $i$ th tree-depth or "scale" (for $i=1,2, \cdots, \log N$ ). Note that $b_{j}^{i}$ represents the $\boldsymbol{R}^{N}$-basis members associated with node $n_{j}^{i}$ while $c_{j}^{i}$ represents the inner product of the signal with the basis vectors in $b_{j}^{i}$. Thus, for the depth-2 decomposition for a Haar wavelet kernel (see Fig. 1(a)), the 5 ON bases corresponding to all permissible subtrees are listed in Fig. 5. Note also that to simplify notation, the subscripts $i, j$ will be dropped where not necessary, thus resulting in node $n$, along with its associated basis and coefficient vectors $b_{n}$ and $c_{n}$, respectively.

- $\boldsymbol{q}_{\boldsymbol{a}}(n)$ : Set of all admissible quantizers for node $n \in T$. The toy example at the end of this section presents some admissible quantizer choices for a particular case.

- $\boldsymbol{Q}_{\boldsymbol{a}}(S)$ : Vector set of all admissible quantizers for the collection of individual leaf nodes of subtree $S=$ $\left\{\boldsymbol{q}_{\boldsymbol{a}}\left(n_{1}\right) \times \boldsymbol{q}_{\boldsymbol{a}}\left(n_{2}\right) \times \cdots \times \boldsymbol{q}_{\boldsymbol{a}}\left(n_{L}\right)\right\}$, where $\left\{n_{1}, n_{2}, \cdots\right.$ $\left.n_{L}\right\} \in \tilde{S}$, is the complete set of leaves or terminal nodes of $S$.

- $D_{q}(n), R_{q}(n)$ : Quantization distortion and bit rate, respectively, for WP coefficient vector $c_{n}$ of node $n$ using quantizer $q \in \boldsymbol{q}_{\boldsymbol{a}}(n)$. Note that we assume completely 


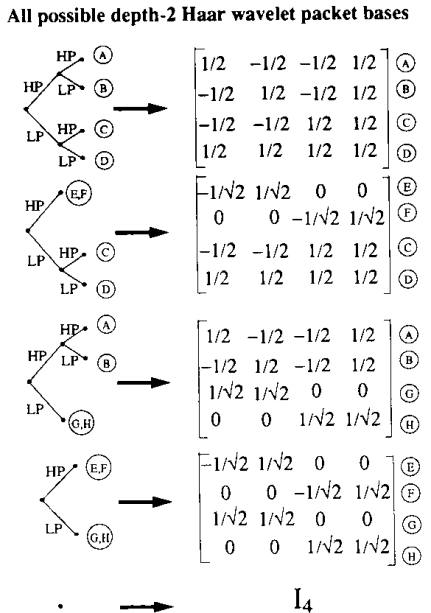

Fig. 5. The five possible orthonormal wavelet packet bases for a depth-2 tree using the Haar wavelet kernel. Note that HP refers to the high-pass "difference" filter $H_{0}(z)=1 / \operatorname{sqrt}(2)\left[1-z^{-1}\right]$ and LP refers to the low-pass "sum" filter $H_{1}(z)=1 / \operatorname{sqrt}(2)\left[1+z^{-1}\right]$.

arbitrary quantizers (including VQ), and completely arbitrary (entropy) coding schemes. The only assumption we invoke is that the nodes be coded independently and that the distortion measure be additive.

- $D_{Q}(S), R_{Q}(S)$ : distortion and rate, respectively, associated with coding subtree (or wavelet packet) $S$ using quantizer $Q \in \boldsymbol{Q}_{\boldsymbol{a}}(S)$. In our case, they are both linear tree functionals; i.e., the total tree rate and distortion values are, respectively, additive over those of the leaf nodes: The total distortion $\left(D_{Q}(S)\right)$ is $\sum_{n \in \tilde{S}} D_{q}(n)$ and the total rate $\left(R_{Q}(S)\right)$ is $\sum_{n \in \bar{S}} R_{q}(n)$.

The problem to solve, then, is that of finding, given a total budget of $R_{\text {budget }}$ to code $M$ independent signal blocks, that sequence of WP "best-bases" $S_{i}^{*} \preceq T$ (for $i=1,2, \cdots, M$ ) together with their associated optimal quantizers $Q_{i}^{*} \in \boldsymbol{Q}_{\boldsymbol{a}}\left(S_{i}^{*}\right)$ which minimize the global coding distortion. Note that orthonormality at the block boundaries can be preserved either by a periodic extension of the blocks at the boundaries or by using ON boundary filters [16]. Stated mathematically, this boils down to determining $D_{\min }=\sum_{i=1}^{M} D_{Q_{i}^{*}}\left(S_{i}^{*}\right)$, where,

$$
D_{Q_{i}^{*}}\left(S_{i}^{*}\right)=\min _{S_{i} \preceq T}\left[\min _{Q_{i} \in \boldsymbol{Q}_{\boldsymbol{a}^{\left(S_{i}\right)}}} D_{Q_{i}}\left(S_{i}\right)\right]
$$

such that

$$
R_{\text {total }}=\sum_{i=1}^{M} R_{Q_{i}^{*}}\left(S_{i}^{*}\right) \leq R_{\text {budget }}
$$

where $R_{\text {budget }}$ is the given bit budget constraint.

\section{TOY EXAMPLE}

As an example, suppose we want to find the best wavelet packet basis corresponding to the Haar wavelet kernel for an input signal $s=[109,23,-98,13]$, with one block and dimension 4 , i.e., $N=4, M=1$. for a coding budget of 21 bits.
Assume the following classes of admissible quantizers for each tree level: we have three grades of uniform quantizers (coarse, medium, and fine) having step sizes of 16,4 , and 1 resp., or equivalently, a granularity of 16,64 , and 256 levels (using 4, 6, and 8 bits) respectively, assuming a quantizer dynamic range from -128 to +128 . As shown in Fig. 4, for convenience, the tree scales are denoted by the labels A, B, and C. At full tree-depth $C$, the quantizers 1,2 , and 3 denote the fine, medium, and coarse scalar quantizers for each of the 4 wavelet packet coefficients $\mathrm{C} 1-\mathrm{C} 4$. At depth $\mathrm{B}$, the quantizers 1,2 , and 3 denote the [fine, fine], [medium, medium] and [coarse, coarse] combination of quantizers applied independently to each of the wavelet packet coefficients B1 and B2. At the tree root $\mathrm{A}$, similar vectors of the three different grades of scalar quantizers are available to code the 4-D coefficient. Assume an MSE distortion criterion.

Note that the wavelet packet coefficients are the inner products of the input signal with the respective basis vectors (see Fig. 4):

$$
\begin{aligned}
& c_{1}^{0}=[109,23,-98,13] \\
& c_{1}^{1}=[-60.81,78.49] ; \quad c_{2}^{1}=[93.34,-60.1] \\
& c_{1}^{2}=[98.5] ; c_{2}^{2}=[12.5] ; \\
& c_{3}^{2}=[-108.5] ; \quad c_{4}^{2}=[23.5] .
\end{aligned}
$$

Fig. 6 shows the rate-distortion curves for all possible basis subtrees in our example, for the permissible quantization choices. Thus, for example, $c_{1}^{0}=[109,23,-98,13]$ would be quantized to $[108,24,-96,12]$ (for a total squared-error distortion of 7.0) with the medium grade (step-size 4) quantizer, and so on.

\section{FAST SOLUTION}

We solve the constrained problem of (2) by converting it to an unconstrained problem using Lagrange multipliers. This section spells out the unconstrained approach, and explains how our problem is a hierarchical extension of that presented in [4]. A fast pruning algorithm is used to remove suboptimal subtrees that would not otherwise have been eliminated if we had resorted to a "flattened" version of our problem to emulate that solved in [4]. Solving the unconstrained problem for different positive values of the Lagrange multiplier results in the tracing out of convex hull points of the rate-distortion curve. The optimal convex hull point we solicit is that with the minimum distortion while not exceeding the given rate budget.

\section{A. Unconstrained Optimization Approach}

Instead of solving the constrained optimization problem (2), let us consider the following unconstrained formulation. Let us introduce, for each signal block, the Lagrangian cost functional corresponding to the Lagrange multiplier $\lambda \geq 0$, for basis subtree $S \preceq T$ and subtree quantizer set $Q \in \bar{Q}_{\boldsymbol{a}}(S)$

$$
\begin{aligned}
J_{Q}(S, \lambda) & \triangleq D_{Q}(S)+\lambda R_{Q}(S) \\
& =\sum_{n \in \tilde{S}}\left[D_{q}(n)+\lambda R_{q}(n)\right]
\end{aligned}
$$



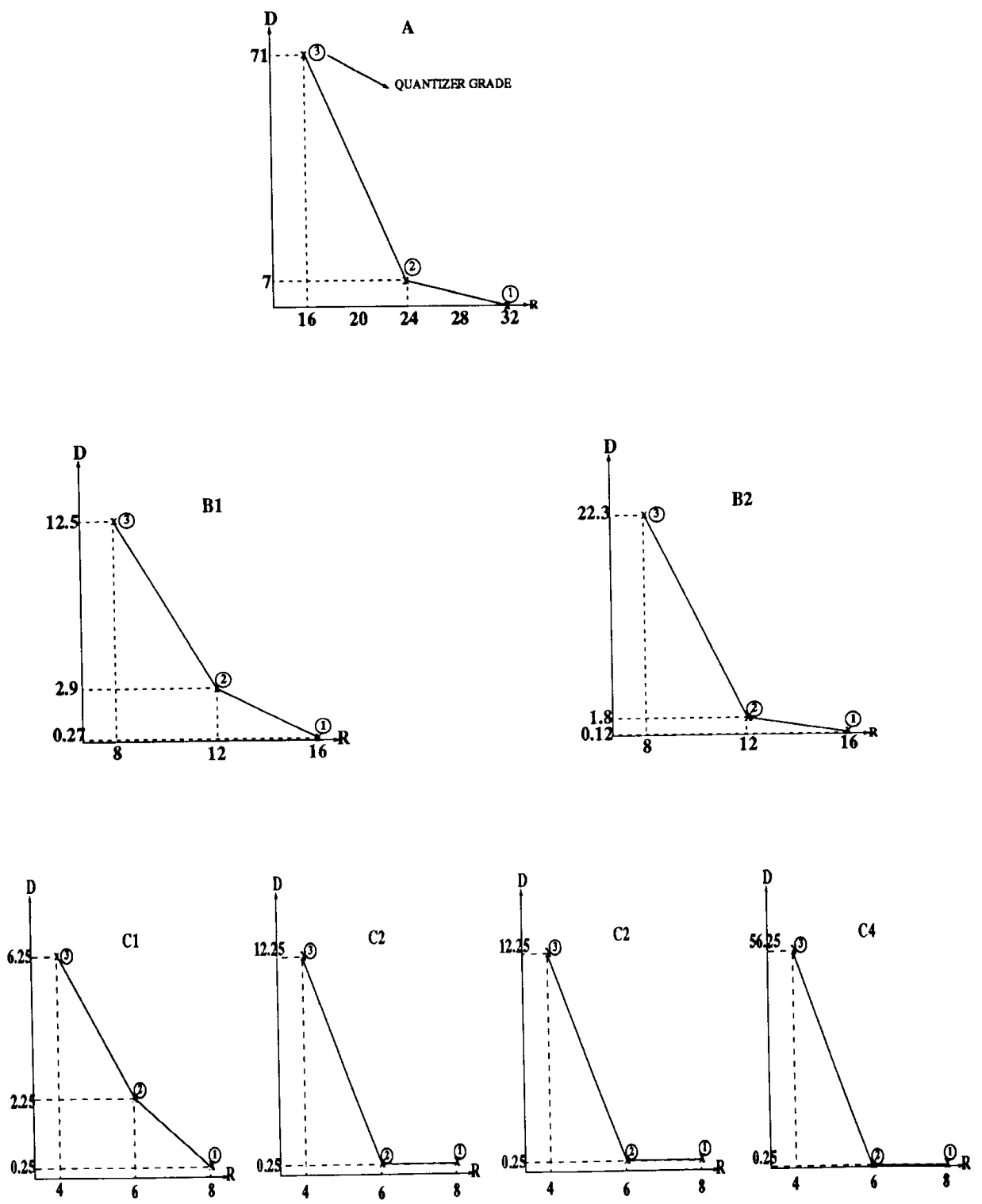

Fig. 6. Toy example R-D curve at tree-depth 0 (A), depth 1 (B1, B2), and depth $2(\mathrm{C} 1-\mathrm{C} 4)$. Note that B1 refers to the HPF output, and B2 to the LPF, and similarly $(\mathrm{C} 1, \mathrm{C} 2)$ are the HPF/LPF outputs of the B1 input, and $(\mathrm{C} 3, \mathrm{C} 4)$ of the B2 input. Sec Fig. 5.

where the last equation is written in terms of the leaf nodes of the subtree.

We now develop, by a simple extension of Theorem 1 in [4] to include the ensemble of WP bases $S \preceq T$ as well as their associated quantizers $Q(S) \in \boldsymbol{Q}_{\boldsymbol{a}}(S)$, an equivalent unconstrained problem. This formulation is attractive because it decomposes the original problem into independent parallel optimizations for each signal block. Mathematically stated, for a fixed value of $\lambda$, the unconstrained problem specified below is solved for $S_{u}^{*}, Q_{u}^{*}$ that minimize the total Lagrangian subtree cost.

$$
J_{Q_{u}^{*}}\left(S_{u}^{*}, \lambda\right)=\min _{S \preceq T} \min _{Q \in \boldsymbol{Q}_{\boldsymbol{a}}(S)}\left[D_{Q}(S)+\lambda R_{Q}(S)\right]
$$

$$
=\min _{S \preceq T} \sum_{n \in \hat{S}} \min _{q \in \boldsymbol{q}_{\boldsymbol{a}}^{(n)}}\left[D_{q}(n)+\lambda R_{q}(n)\right] .
$$

The constrained optimization problem of (2) for the single block $(M=1)$ case becomes

$$
D_{Q_{r}^{*}}\left(S_{c}^{*}\right)=\min _{S} \min _{Q} D_{Q}(S) \text { s.t. } R_{Q_{c}^{*}}\left(S_{c}^{*}\right) \leq R_{\text {budget }}
$$

while its unconstrained counterpart from (6) is

$$
J_{Q_{u}^{*}}\left(S_{u}^{*}, \lambda\right)=\min _{S} \min _{Q}\left[D_{Q}(S)+\lambda R_{Q}(S)\right] .
$$

The above approach identifies, for a fixed positive $\lambda$, an optimal operating point on the convex hull of the compos- 
ite $\mathrm{R}-\mathrm{D}$ curve for the specified problem. If the original constrained problem happened to have a budget constraint that "hits" one of the convex-hull operating points, then the unconstrained and constrained problems have identical solutions. Mathematically stated, the equivalence is established in the following theorem:

Theorem 1: If $\left(S_{u}^{*}, Q_{u}^{*}\right)$ is the solution of the unconstrained problem of (9) corresponding to some fixed value of $\lambda$, then it is also the solution to the constrained problem of (8) for the particular case of $R_{\text {budget }}=R_{Q_{u}^{*}}\left(S_{u}^{*}\right)$; i.e., for this budget $R_{Q_{u}^{*}}=R_{Q_{c}^{*}}$ and $S_{Q_{u}^{*}}=S_{Q_{c}^{*}}$.

$$
\begin{aligned}
& \text { Proof: } \\
& \qquad \begin{aligned}
J_{Q_{u}^{*}}\left(S_{u}^{*}, \lambda\right) & \leq J_{\lambda}(S, Q) \\
D_{Q_{u}^{*}}\left(S_{u}^{*}\right)+\lambda R_{Q_{u}^{*}}\left(S_{u}^{*}\right) & \leq D_{Q}(S)+\lambda R_{Q}(S) \\
D_{Q_{u}^{*}}\left(S_{u}^{*}\right)-D_{Q}(S) & \leq \lambda\left[R_{Q}(S)-R_{Q_{u}^{*}}\left(S_{u}^{*}\right)\right] \\
D_{Q_{u}^{*}}\left(S_{u}^{*}\right)-D_{Q}(S) & \leq \lambda\left[R_{Q}(S)-R_{\text {budget }}\right]
\end{aligned}
\end{aligned}
$$

Since (13) holds for all $S \preceq T$ and $Q \in \boldsymbol{Q}_{\boldsymbol{a}}(S)$, it certainly holds for the subsets $\bar{S} \preceq T, \overline{\boldsymbol{Q}} \in \boldsymbol{Q}_{\boldsymbol{a}}(\bar{S})$ which satisfy $R_{Q}(S) \leq R_{\text {budget. }}$. That is,

$$
R_{Q}(S) \leq R_{\text {budget }} \quad \text { for } S \in \bar{S}, Q \in \bar{Q} .
$$

Thus from (13) and (14), since $\lambda \geq 0$, we have

$$
D_{Q_{u}^{*}}\left(S_{u}^{*}\right)-D_{Q_{u}}\left(S_{u}\right) \leq 0 \quad \forall S \in \bar{S}, Q \in \overline{\boldsymbol{Q}}
$$

i.e., $\left(S_{u}^{*}, Q_{u}^{*}\right)$ also satisfies the original constrained optimization problem of (8) for the given budget constraint, and $R_{Q_{u}^{*}}=R_{Q_{*}^{*}}$ and $S_{Q_{u}^{*}}=S_{Q_{c}^{*}}$.

Note that the above result implies that if we solve the unconstrained problem of (9) for some $\lambda \geq 0$, and if $R_{\text {budget }}$ of the constrained problem of (8) happens to be $R_{Q_{u}^{-}}\left(S_{u}^{*}\right)$ of the unconstrained problem, then the solutions to both problems are identical.

\section{B. "Flattening" the Problem}

Let us define $X_{i}$ to be the set of all $(R, D)$ operating points corresponding to block $i$ of the signal. Note that $X_{i}$ must include all combinations of permissible WP bases $S_{i} \preceq T$ together with their associated quantization choices $Q_{i} \in Q_{a}\left(S_{i}\right)$.

Thus the "flattened" equivalent of our constrained optimization problem of (2) is

$$
\min _{x_{i} \in X_{i}} \sum_{i} D\left(x_{i}\right)
$$

subject to

$$
\sum_{i} R\left(X_{i}\right) \leq R_{\text {budget }}
$$

For every $S$, there exist $\left|\boldsymbol{Q}_{\boldsymbol{a}}(\boldsymbol{S})\right|$ such $(R, D)$ points; by a simple inductive argument, it can be shown that for a binary tree of depth $d$, there exist about $2^{2^{d}}$ WP bases. Thus although it is possible for us to "flatten" our hierarchical quantization problem and solve it using the method of Shoham and Gersho [4], this is obviously computationally expensive. We solve the

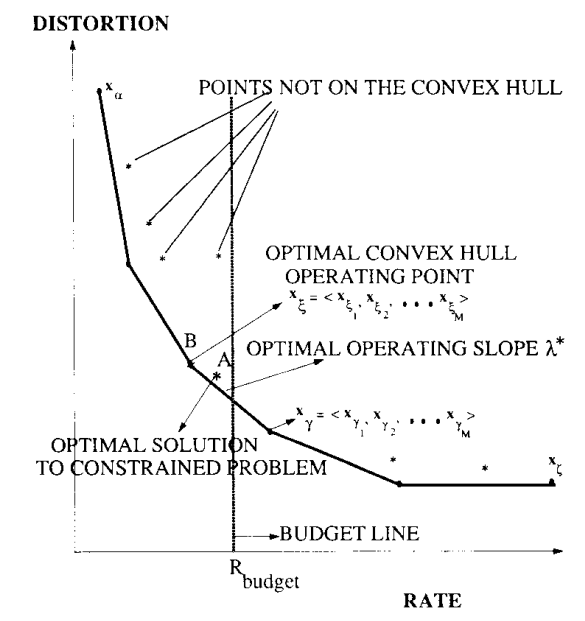

Fig. 7. Composite R-D curve showing convex hull solution to an "inaccessible" problem.

problem instead by employing a fast pruning algorithm to be described in Section V-E.

The flattened version of the problem can however be used to inherit some of the key properties of the unconstrained formulation. Some of the key results inherited from [4], as they apply to our problem, are hence presented as a summary. The unconstrained allocated rate and distortion values $R(\lambda)$ and $D(\lambda)$ are monotonic in the Lagrange multiplier $\lambda$. As $\lambda$ is swept through all positive real numbers, all the convex hull points of the composite $R-D$ curve are traced out. See Fig. 7 for a typical composite $R-D$ curve comprising $M$ signal blocks. Thus $\lambda$ could be interpreted as a quality index as it is swept from 0 (highest rate, lowest distortion) to $\infty$ (lowest rate, highest distortion).

\section{Finding the Optimal $\lambda$}

With $J(\lambda)=D(x)+\lambda R(x)$ representing the Lagrangian subcost associated with operating point $x$ of a typical block (note that the block index $i$ is dropped for convenience) for quality criterion $\lambda$, let us introduce the biased Lagrangian cost $W$ as

$$
\begin{aligned}
W(\lambda) & =W\left(\lambda, x^{*}(\lambda)\right) \\
& =J^{*}(\lambda)-\lambda R_{\text {budget }} \\
& =\left(\min _{x}[D(x)+\lambda R(x)]-\lambda R_{\text {budget }}\right) .
\end{aligned}
$$

Then, following the optimization theory outlined in [17], we have the following result:

Lemma 1: $W(\lambda)$ is a concave $\cap$ function of $\lambda$.

Proof: See Appendix.

Now, if we find the maximum of $W(\lambda)$ over all positive $\lambda$,

$$
W\left(\lambda^{*}\right)=W\left(\lambda^{*}, x^{*}\left(\lambda^{*}\right)\right)=\max _{\lambda \geq 0} W(\lambda)
$$

we have the following result for the unconstrained solution corresponding to the given budget constraint $R_{\text {budget }}$ : 
Theorem 2: $\lambda^{*}$ and $x^{*}\left(\lambda^{*}\right)$ that maximize $W$ in (20) are the optimal convex hull face slope and optimal convex hull operating point, respectively, for the unconstrained optimization problem of (6), for the given budget constraint $R_{\text {budget }}$.

Proof: See Appendix.

Thus the above result gives the condition on the desired operating quality slope which solves the flattened version of our original problem. By "unflattening" the above result, we now develop the unconstrained solution to our best wavelet packet basis and optimal quantization choice problem. The optimal slope $\lambda^{*}$ is the solution to

$$
\begin{aligned}
& W\left(\lambda^{*}\right)=W\left(\lambda^{*}, x^{*}\left(\lambda^{*}\right)\right) \\
& =\max _{\lambda \geq 0}\left(\left[\min _{S} \min _{Q} J_{Q}(S, \lambda)\right]\right. \\
& =\max _{\lambda \geq 0}\left(\left[\min _{S}\left\{\sum_{n \in \tilde{S}} \min _{q}\left[D_{q}(n)+\lambda R_{q}(n)\right]\right\}\right]\right. \\
& \left.-\lambda R_{\text {budget }}\right) .
\end{aligned}
$$

The trivial generalization to the multiple block allocation problem $(M>1)$ is then

$$
\begin{gathered}
W\left(\lambda^{*}\right)=\max _{\lambda \geq 0}\left(\sum_{i=1}^{M}\left[\min _{S_{i}}\left\{\sum_{n \in \tilde{S}_{i}} \min _{q}\left[D_{q}(n)+\lambda R_{q}(n)\right]\right\}\right]\right. \\
\left.-\lambda R_{\text {budget }}\right) .
\end{gathered}
$$

This is then the unconstrained optimization formulation to our problem, which can be dissected into independent fast individual optimizations. Thus (24) can be dissected as follows into 3 optimization operations

$$
\begin{aligned}
& \operatorname{mix}_{\lambda}^{(3)}\left(\sum _ { \text { blocks } } \left[\underset { \text { mubtrees } } { ( 2 ) } \left\{\sum_{\text {leaves }} \underset{\text { quantizers }}{\stackrel{(1)}{\min }}[D \text { (node) }\right.\right.\right. \\
& \left.+\lambda R(\text { node })]\}]-\lambda R_{\text {budget }}\right) \text {. }
\end{aligned}
$$

1) At first (innermost minimization), select the quantizers for each leaf node of the fixed $W P$ basis $S$ which minimize $D+\lambda R$ for fixed operating slope $\lambda$, independently for every block.

2) Next (outer minimization), find the best WP basis subtree $S$ (from the entire ON library independently for each block again for the fixed operating slope $\lambda$. A fast dynamic programming based split/merge pruning operation will be used to accomplish this (see Section V-E).

3) Finally (outermost maximization), determine the optimal slope $\lambda^{*}$ for the given bit budget $R_{\text {budget }}$ as the maximum of $W(\lambda)$. Lemma 1 facilitates the use of fast search methods for finding the optimal $\lambda^{*}$ in an iterative fashion (see Section VI-B).

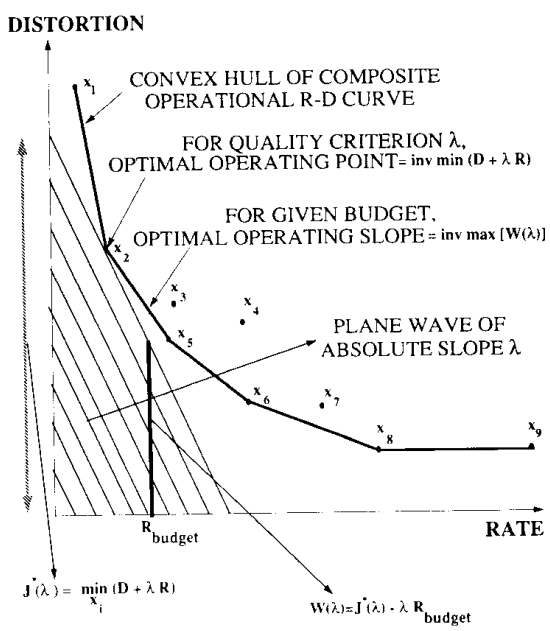

Fig. 8. Geometric interpretation of the problem

\section{Geometric Interpretation}

One insight to be made into the unconstrained optimization problem is that of a geometric approach. It can be shown that the optimal operating point on the $\mathrm{R}-\mathrm{D}$ plane for each leaf node of the tree $T$ for a given slope $\lambda$ is that point in the collection of R-D points which is first "impinged upon" by a "plane-wave" of slope $-\lambda$ emanating from the fourth quadrant of the $\mathrm{R}-\mathrm{D}$ plane towards the $\mathrm{R}-\mathrm{D}$ curve in the first quadrant. This is because the Lagrangian cost $J$ associated with any admissible operating point can be interpreted as the $y$-intercept of the straight line of slope $-\lambda$ passing through that point on the operational rate-distortion plane. See Fig. 8 . The minimum Lagrangian function (minimum $y$-intercept) is obviously achieved for that point which is "hit" first by the plane wave of absolute slope $\lambda$ impinging on the rate-distortion curve. Note also from Fig. 8 that the biased Lagrangian function $W(\lambda)$ can be interpreted as the intercept, on the budget constraint line, of the straight line of slope $-\lambda$ tangent to the convex hull of the $\mathrm{R}-\mathrm{D}$ curve. This geometric interpretation of the problem makes properties like monotonicity, existence of singular slope values, the concavity of $W$, and the R-D convex hull solution both intuitively pleasing and easy to show using straight-line geometry, though a more rigorous algebraic proof is provided in the appendix.

\section{E. Finding the Best Basis Subtree}

The difference between the flattened approach and the hierarchical approach is in the search for the best basis subtree. While a flattened approach entails an exhaustive search of the entire ON library of WP's in a "brute force" manner, the hierarchical approach uses a fast split/merge "pruning" algorithm to determine the best basis.

A fast dynamic programming technique, similar to the CMQW approach [1], is feasible here due to the orthonormal property of the WP basis family, that enables the signal space spanned by an arbitrary subtree rooted at internal node $n$ of the tree to be identical to the space spanned by the twin subtrees 
rooted at the two branches emanating from node $n$. To be specific, let $n_{\text {parent }}=n_{j}^{i}$, i.e., $n_{\text {parent }}$ is the $j$ th node of the $i$ th scale of the tree $T$. Its two children are $n_{\text {child } 1}=n_{2 j-1}^{i+1}$ and $n_{\text {child } 2}=n_{2 j}^{i+1}$. Then, because of the ON property, the subtrees rooted at $n_{\text {child1 }}$ and $n_{\text {child2 }}$ cover disjoint halves of the $R^{N / 2^{i}}$ signal space spanned by their parent node $n_{\text {parent }}$.

This allows a direct quantitative one-to-one comparison between the $N / 2^{i}$ basis coefficients $\left\{c_{j}^{i}\right\}$ associated with the basis subset $\left\{b_{j}^{i}\right\}$ of node $t$ with the $\left(2 \times\left(N /\left(2^{(i+1)}\right)\right)\right)$ coefficients $\left\{\left\{c_{2 j-1}^{i+1}\right\},\left\{c_{2 j}^{i+1}\right\}\right\}$ associated with the basis subsets $\left\{b_{2 j-1}^{i+1}\right\}$ and $\left\{b_{2 j}^{i+1}\right\}$ of nodes $t_{1}$ and $t_{2}$ resepectively. The "split/merge" decision will be based on the option which leads to a cheaper Lagrangian cost, as spelled out in Fig. 3.

Assume known the optimal subtree from node $n$ "onwards" to the full tree-depth $\log N$. Then by Bellman's optimality principle [18], we know all surviving paths passing through node $n$ must invoke this same optimal "finishing" path. There are two contenders for the "surviving path" at every node of the tree, the parent and its children, with the winner having the lower Lagrangian cost. Using this, we begin at the complete tree-depth $n=\log N$ and work our way towards the root of the tree, using the above split/merge criterion at each node. This decision (or "path") is remembered and used to determine the best path when applying the same pruning criterion on the branches, which process is repeated till the root is encountered. At this point, the best basis is known.

\section{COMPLETE Algorithm}

The stage is now set to integrate the results of the previous two sections to formulate the optimal algorithm. This will be done in two phases. First, the flowchart of the optimal algorithm for a given operating slope $\lambda$ will be detailed, followed by a description of the hunt for the optimal operating slope $\lambda^{*}$. Note that the algorithm is applied independently on each signal block to determine the best wavelet packet basis corresponding to that subblock (for a given $\lambda$ ).

\section{A. Initialization}

Prior to the actual pruning operation, a one-time fixed cost of gathering the statistics enlisted in Steps 1 and 2 below must be endured. Associated with every node $n_{j}^{i}$ of $T$ is a data structure of the form: $\left\{\hat{R}_{j}^{i}, \tilde{D}_{j}^{i}, \hat{J}_{j}^{i}\right.$, split $\left.\left(n_{j}^{i}\right)\right\}$. The first three members refer to the rate, distortion, and Lagrangian cost associated with the optimal (for a given $\lambda$ ) subtree from $n_{j}^{i}$ onwards, i.e., the optimal subtree rooted at $n_{j}^{i}$, while the last member of the data structure, split $\left(n_{j}^{i}\right)$, is a binary variable whose meaning (yes or no) reflects the decision of whether or not it is optimal to split the node into its children branches.

Step 1: Generate the coefficients $\left\{c_{j}^{i}\right\}$ for the entire WP family.

Step 2: Gather the given quantizer set dependent $\left(R_{q}(n)\right.$, $\left.D_{q}(n)\right)$ values for all the nodes $n \in T \quad \forall q \in \boldsymbol{q}_{\boldsymbol{a}}(n)$, to generate the $R$ versus $D$ points for each node.

Phase I: Optimality For A Given Operating Slope: Phase I of the algorithm is run for a given slope value $\lambda$, and could be considered a subroutine called by the Phase II, described later in the section, for the fixed budget allocation problem:

Step 3: For the current $\lambda$, populate all the nodes $n$ of the tree with their minimum Lagrangian costs $J_{n}(\lambda)$, or equivalently $J_{j}^{i}(\lambda)$ when referring to the $j$ th node at scale $i$ : i.e., $J_{n}(\lambda)=\min _{q}\left[D_{q}(n)+\lambda R_{q}(n)\right]$.

Step 4: Initialize $i \leftarrow d$, where $d=\log N$ is the maximum signal block tree-depth. For $n=n_{j}^{d}$, if $q_{n}^{*}$ is the value of $q_{n}$ that minimizes $J_{n}(\lambda)$ initialize

$$
\begin{array}{ll}
\tilde{R}_{j}^{d} \leftarrow R_{j}^{d} & \text { (where } \left.R_{j}^{d}=R_{q_{n}^{*}}(n)\right) \\
\tilde{D}_{j}^{d} \leftarrow D_{j}^{d} & \text { (where } \left.D_{j}^{d}=D_{q_{n}^{*}}(n)\right) \\
\tilde{J}_{j}^{d} \leftarrow J_{j}^{d} . &
\end{array}
$$

Step 5: $i \leftarrow i-1$. If $i<0$, go to Step 8 .

Step 6: $\forall j=1,2, \cdots, 2^{i}$ at the $i$ th tree level:

$$
\begin{aligned}
& \text { if } J_{j}^{i}(\lambda)<\tilde{J}_{2 j-1}^{i+1}(\lambda)+\tilde{J}_{2 j}^{i+1}(\lambda), \\
& \text { then }\left\{\operatorname{split}\left(n_{j}^{i}\right) \leftarrow \mathrm{NO} ; \tilde{R} \leftarrow R_{j}^{i} ; \tilde{D}_{j}^{i} \leftarrow D_{j}^{i} ; \tilde{J}_{j}^{i} \leftarrow J_{j}^{i}\right\} \\
& \operatorname{else}\left\{\operatorname{split}\left(n_{j}^{i}\right) \leftarrow \mathrm{YES} ; \tilde{R}_{j}^{i} \leftarrow R_{2 j-1}^{i+1}+R_{2 j}^{i+1} ;\right. \\
& \left.\quad \tilde{D}_{j}^{i} \leftarrow D_{2 j-1}^{i+1}+D_{2 j}^{i+1} ; \tilde{J}_{j}^{i} \leftarrow J_{j-1}^{i+1}+J_{2 j}^{i+1}\right\} .
\end{aligned}
$$

Step 7: Go to Step 5.

Step 8: Starting from the root $n_{0}$, and using, in a linked-list fashion, the node data-structure element split (node), selected optimally for all the nodes of $T$, carve out the optimal subtree $S^{*}(\lambda)$ and its associated optimal quantizer choice $Q^{*}(\lambda) \in$ $\boldsymbol{Q}_{\boldsymbol{a}}\left(S^{*}(\lambda)\right)$. Also readily available at the data-structure for root node $n_{0}$ are $R_{Q^{*}}\left(S^{*}\right)=\tilde{R}_{1}^{0}$ and $D_{Q^{*}}\left(S^{*}\right)=\tilde{D}_{1}^{0}$, the rate and distortion of the optimal subtree $S^{*}(\lambda)$.

Note that it is possible to directly incorporate into the pruning algorithm the cost of segmentation (in terms of overhead bits of the subtree map to be sent), if an a priori maprepresentation scheme is available. For example, if the subtree structure costs one bit per merge decision, this bit could be included in the Lagrangian cost comparison of the children nodes with the parent node in Step 6 of the Phase I algorithm outlined above. However, in our generalized algorithm, no a priori map-coding scheme is assumed, and $R_{\text {budget }}$ is used for pure coding expenditure without any overhead expenses, which may be minimized using entropy coding of the treemap if necessary. In our image coding applications, we found the overhead to represent a negligible proportion of the total budget. For example, for an image block size of $128 \times 128$ using a WP decomposition of depth 4 (resulting in $8 \times 8$ leaf nodes), the cost of sending the tree map is less than 0.000625 bits per pixel, certainly negligible for most applications!

\section{B. Finding the Optimal Operating Slope}

The problem of picking the optimal slope value for a given budget criterion $R_{\text {budget }}$ is the subject of discussion in Phase II of the algorithm; the iterative invocations of the Phase I subroutine in search of the optimal operating slope $\lambda_{\text {opt }}$ for the given $R_{\text {budget }}$, will be described in this section. 
As was shown in Section $\mathrm{V}$, due to the concavity of $W(\lambda)$ in $\lambda$ (see Fig. 9), and since our optimal operating slope $\lambda^{*}$ is $\max ^{-1}(W)$, we can find our optimal operating point using a fast convex search algorithm like Newton's method or bisection methods [19]. Equivalently stated, we are interested in the zero-crossing of the derivative of $W, \partial W / \partial \lambda$. Recall that

$$
W(\lambda)=\left(\sum_{i} \min _{x_{i} \in X_{i}}\left[D\left(x_{i}\right)+\lambda R\left(x_{i}\right)\right]\right)-\lambda R_{\text {budget }}
$$

which implies that, at nonsingular values of $\lambda$,

$$
\partial W / \partial \lambda=\sum_{i} R_{i}^{*}(\lambda)-R_{\text {budget }}
$$

where $R_{i}^{*}(\lambda)$ is the rate associated with the optimal subtree/quantizer choice for block $i$. Due to the discrete nature of our problem, $\lambda$ is singular at only a finite number of points (see Fig. 9). Also, as was developed in Theorem 2 (see appendix), the optimal slope $\lambda^{*}$ which maximizes $W$ corresponds to a singular value. From (25), at nonsingular values of $\lambda<\lambda^{*}$, we have $\sum_{i} R_{i}^{*}(\lambda)>R_{\text {budget }}$, while for nonsingular values of $\lambda>\lambda^{*}, \sum_{i} R_{i}^{*}(\lambda) \leq R_{\text {budget }}$. This then leads to the iterative fast convex search algorithm to be described.

As with most iterative solutions, the choice of a good initial operating point is the key to a fast convergence. Assume we have judiciously chosen two values of $\lambda, \lambda_{l}$ and $\lambda_{u}$ with $\lambda_{l} \leq \lambda_{u}$ which satisfy the relation:

$$
\sum_{i} R_{i}^{*}\left(\lambda_{u}\right) \leq R_{\text {budget }} \leq \sum_{i} R_{i}^{*}\left(\lambda_{l}\right)
$$

Note that failure to find any $\lambda_{l}, \lambda_{u}$ which satisfy the above inequalities means that the given problem is unsolvable; i.e., the $R_{\text {budget }}$ is inconsistent with the given sets of quantizers. A conservative choice for a solvable problem would be $\lambda_{l}=$ $0, \lambda_{u}=\infty$.

Phase II: Iterating towards the optimal operating point: Now the following "main" algorithm can be used to iteratively call the "subroutine" algorithm of the previous section:

Step 1: Pick $\lambda_{l} \leq \lambda_{u}$ such that

$$
\sum_{i} R_{i}^{*}\left(\lambda_{u}\right) \leq R_{\text {budget }} \leq \sum_{i} R_{i}^{*}\left(\lambda_{l}\right) .
$$

If the inequality above is an equality for either slope value, stop. We have an exact solution. Otherwise, proceed to Step 2.

Step 2: $\lambda_{\text {next }} \leftarrow \mid\left(\sum_{i}\left[D_{i}^{*}\left(\lambda_{l}\right)-D_{i}^{*}\left(\lambda_{u}\right)\right]\right) /\left(\sum_{i}\left[R_{i}^{*}\left(\lambda_{l}\right)-\right.\right.$ $\left.\left.R_{i}^{*}\left(\lambda_{u}\right)\right]\right) \mid+\epsilon$, where $\epsilon$ is a vanishingly small positive number picked to ensure that the lower rate point is picked if $\lambda_{\text {next }}$ is singular slope value.

Step 3: Run the Phase I optimal algorithm for $\lambda_{\text {next }}$.

$$
\begin{aligned}
& \Rightarrow \text { if }\left\{\sum_{i} R_{i}^{*}\left(\lambda_{\text {next }}\right)=\sum_{i} R_{i}^{*}\left(\lambda_{u}\right)\right\} \text {, then stop. } \lambda^{*}=\lambda_{u} \\
& \Rightarrow \text { else if }\left(\sum_{i} R_{i}^{*}\left(\lambda_{\text {next }}\right)>R_{\text {budget }}\right), \lambda_{l}-\lambda_{\text {next }} .
\end{aligned}
$$

Go to Step 2 .

$$
\Rightarrow \text { else } \lambda_{u} \leftarrow \lambda_{\text {next }} \text {. Go to Step } 2 \text {. }
$$

if $\lambda$ is non-singular,

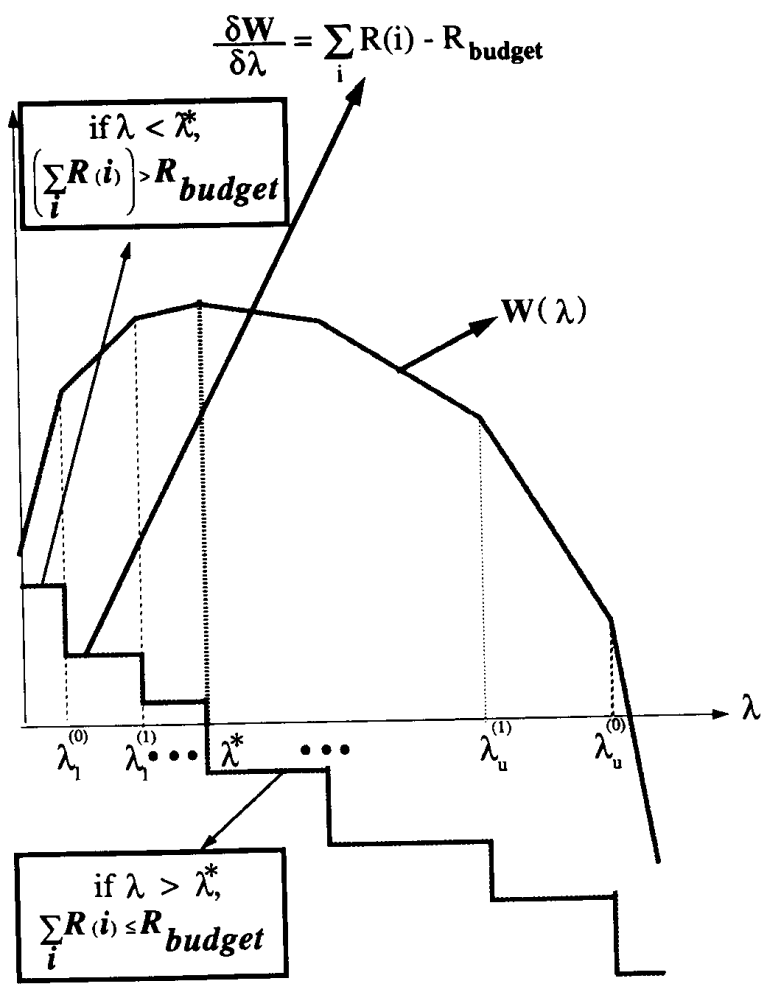

Fig. 9. Concavity of the biased Lagrangian functional $W(\lambda)$ and mathematical justification for fast bisection algorithm.

Thus starting from a known initial interval engulfing the desired operating slope, the search intervals are made successively smaller, exploiting the convex relationship of both global rate and global distortion with respect to the operating slope $\lambda$ until convergence is achieved. In Step 2 above, the convexity property is exploited in tightening either the upper or the lower bound at the $(i+1)^{t h}$ iteration to $\lambda^{(i+1)}=$ $\mid[(\Delta D) /(\Delta R)]^{(i)}$, which provides a tighter bound around $\lambda^{*}$ than available at the $i^{t h}$ iteration.

\section{TOY EXAMPLE}

See Fig. 10 for a plot of the convex hull to the operational rate-distortion curve for the given problem. Shown explicitly are the optimal quantizer and the best basis choice for each operating point, which corresponds to singular values of $\lambda$, whose sweep for 0 to $\infty$ results in the tracing out of all convex hull points. The budget constraint line of 21 bits is obviously an inaccessible convex hull solution, and one has to settle for the convex hull operating point using 20 bits. Note also the nonmonotonic nature of the sequence of the depths of the best bases subtrees as one sweeps $\lambda$ through all positive real numbers.

Let us first show an example of how the Phase I algorithm works for $\lambda=10$ (to pick a nice number) and show how it leads to the lowest quality convex hull point of Fig. 10, which is picked for all values of $\lambda>5.43$ : 


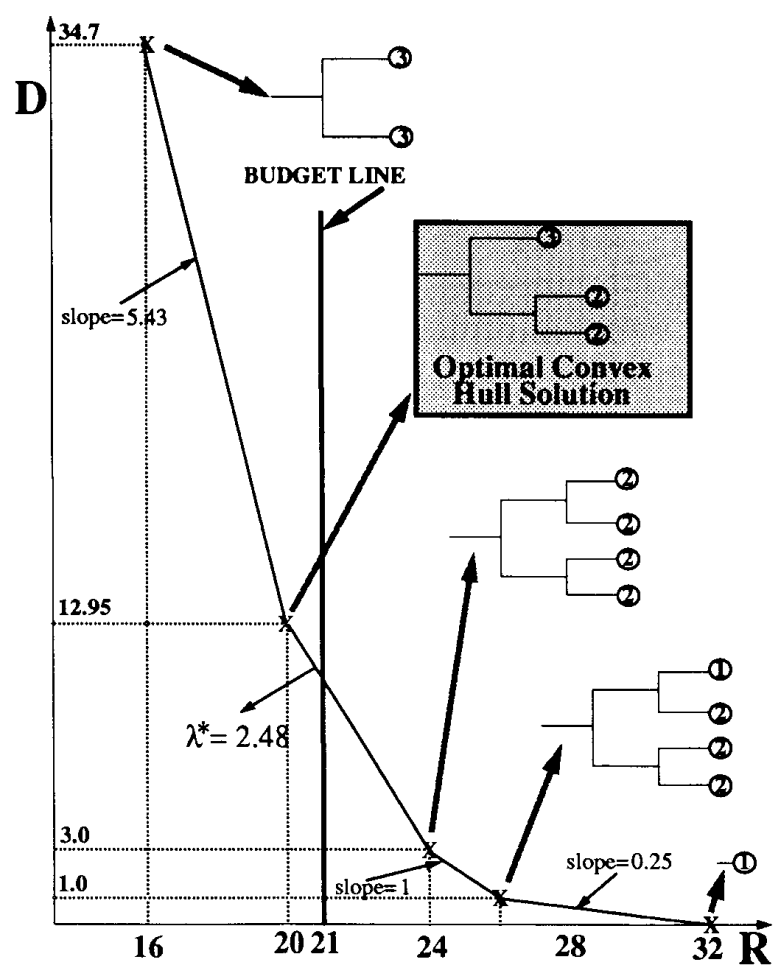

Fig. 10. Composite R-D curve for toy example shown with best basis and best quantizer choices for all convex-hull points, and with optimal tree structure for the given budget constraint. Note the nonmonotonicity of the $\mathrm{R}-\mathrm{D}$ characteristics with tree depth, i.e., nonconformance with the Chou $e$ al. [6] assumptions.

1) Populate the tree with the minimum of all the Lagrangian cost functionals for $\lambda=10$ as outlined in Phase I of the algorithm for each node $\mathrm{A}, \mathrm{B} 1, \mathrm{~B} 2, \mathrm{C} 1, \mathrm{C} 2, \mathrm{C} 3$, and $\mathrm{C} 4$ to get:

$J_{A}=231$ (achieved with quantizer $Q 3$ )

$J_{B 1}=92.5(Q 3) ; \quad J_{B 2}=102.3(Q 3)$

$J_{C 1}=46.25(Q 3) ; \quad J_{C 2}=52.25(Q 3):$

$J_{C 3}=52.25(Q 3) ; \quad J_{C 4}=60.25(Q 2)$.

2)

Initialize $i=2 ; \quad J_{C 1}=46.25: \quad \tilde{J}_{C 2}=52.25$

$$
\tilde{J}_{C 3}=52.25: \quad \tilde{J}_{C 4}=60.25 \text {. }
$$

3) $i=1$; Since $J_{B 1}<\tilde{J}_{C 1}+\tilde{J}_{C 2}$ split $(B 1) \leftarrow \mathrm{NO}$

$$
\begin{aligned}
& J_{B 2}<\tilde{J}_{C 3}+\tilde{J}_{C 1} . \operatorname{split}(B 2) \leftarrow \mathrm{NO} \\
& \tilde{J}_{B 1}=J_{B 1} ; \quad \tilde{J}_{B 2}=J_{B 2} .
\end{aligned}
$$

4) $i=0 ;$ Since $J_{A}>\tilde{J}_{B 1}+\tilde{J}_{B 2}, \operatorname{split}(A) \leftarrow \mathrm{YES}$

$$
\tilde{J}_{A}=\tilde{J}_{B 1}+\tilde{J}_{B 2} \text {. }
$$

We thus have our optimal basis subtree (with quantizer choice) for this value of $\lambda$, as shown as the lowest rate convex hull point of Fig. 10. We now explain in detail the search for $\lambda^{*}$ for the toy problem with a coding budget $R_{\text {budget }}=21$ bits. Refer to Fig. 10.

$$
\begin{aligned}
1) \text { Intialize } \lambda_{l}^{(0)} & =0 ; & & \lambda_{u}^{(0)}=\infty . \\
R^{*}\left(\lambda_{u}\right) & =32 ; & & R^{*}\left(\lambda_{l}\right)=16 .
\end{aligned}
$$

$$
\begin{gathered}
\lambda_{\text {next }}=\frac{34.7-0}{32-16}+\epsilon=2.17+\epsilon \\
\Rightarrow \lambda_{l}^{(1)}=2.17: \quad \lambda_{u}^{(1)}=\infty . \\
\lambda_{\text {next }}=\frac{34.7-3.0}{24-16}+\epsilon=3.96+\epsilon \\
R^{*}\left(\lambda_{\text {next }}\right)=20<R_{\text {budget }}=21: \\
\Rightarrow \lambda_{l}^{(2)}=2.17: \quad \lambda_{u}^{(2)}=3.96 . \\
\lambda_{\text {next }}=\frac{12.95-3.0}{24-20}+\epsilon=2.48+\epsilon \\
R^{*}\left(\lambda_{\text {next }}\right)=R^{*}\left(\lambda_{u}\right)=20 .
\end{gathered}
$$

We have converged! $\Rightarrow \lambda^{*}=2.48 ; D\left(\lambda^{*}\right)=12.95 ; R\left(\lambda^{*}\right)=$ 20 .

\section{IMAGE CODING APPLiCATIONS}

We describe two image compression applications, one a quadtree segmentation using a 2-D DCT basis family in a "hierarchical" JPEG [11] coding environment, and the other involving wavelet packets using the Daubechies D4 [20] set of wavelet filters.

\section{A. DCT Quadtree Application}

The framework of a quadtree structure using a family of DCT bases was used to select the best basis. The coding was performed in a JPEG-like environment using an MSE distortion criterion. In order to have the quantizers adapt to the signal's nonstationarities, classified quantizers were chosen as the admissible set, similar to the VQ application of [21]. Four quantizer classes were used, optimized for 1) "typical" image blocks with low frequencies weighted much higher than the perceptually less sensitive higher frequencies, like the JPEG suggested matrix; (2) horizontal edges; (3) vertical edges; and (4) image blocks with a "white" frequency spectrum, showing no discernible favoritism toward any specific orientation.

\section{B. Coding Description and Simulation Results}

In keeping with perceptual requirements [22], the admissible classes of quantizers described above were constructed for each of three hierarchical levels of the DCT basis tree: $4 \times 4$, $8 \times 8$, and $16 \times 16$ blocks. This is equivalent to a parallel pruning of $16 \times 16$ independent subblock trees of maximum depth 3 . The optimal pruning algorithm of Section VI was used, and "pseudo-JPEG" coding algorithms were followed for the non $-8 \times 8$ blocks, ${ }^{3}$ i.e., DCT transformation, quantization using classified quantizers, zigzag scanning, and Huffman coding of the zero runlengths. Fig. 11 shows comparisons of the adaptive DCT-quadtree coder versus the baseline JPEG coder plotting the peak signal-to-noise-ratio (PSNR) defined as $10 \log _{10}\left[255^{2} /(M S E)\right]$ versus bpp (bits per pixel) for some typical test images used in the image processing community. The results are compared with a typical "static" JPEG coder [11].

In formulating the pseudo-JPEG algorithm for the non$8 \times 8$ blocks, the same default Huffman coding table was

\footnotetext{
${ }^{3}$ The standard JPEG algorithm is applicablc only to $\delta \times \delta$ blocks.
} 

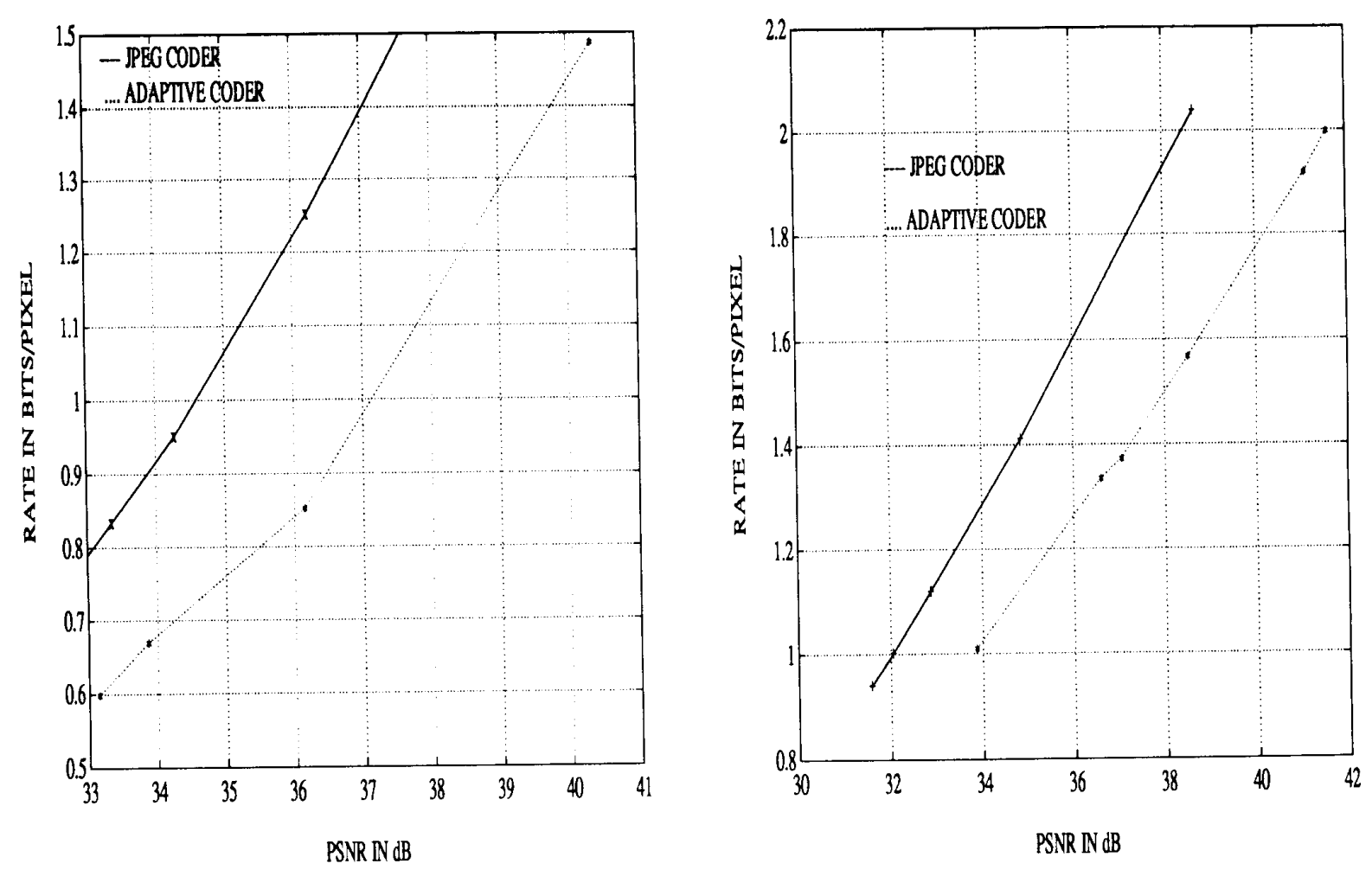

Fig. 11. Comparison of adaptive depth-3 block DCT basis quadtree coding scheme with nonadaptive JPEG coding scheme for the "Barbara" and "mit" images.

used as outlined in the baseline JPEG specification for $8 \times$ 8 blocks. For typical test images, we found that in our coding environment, the adaptive DCT-based quadtree coder outperforms the standard static JPEG coder by about 1.5-2 dB at typical bit rates, or alternatively, by a $15 \%-25 \%$ reduction in bit rate at typical PSNR values. For difficult images like "mit" and "Barbara," our adaptive scheme outperforms JPEG by over $2 \mathrm{~dB}$ at fixed bit rate, or equivalently about $25 \%-35 \%$ compression advantage at fixed SNR, over an entire range of bit rates of interest. Fig. 12 shows a subjective comparison of the optimal adaptive JPEG quadtree segmentation scheme and the static standard JPEG scheme, using a blowup of a portion of the "mit" image to help illustrate the point.

\section{Wavelet Packet Application}

Two-dimensional separable length-8 orthonormal Daubechies D4 wavelet sets of filters [20] were used for this application. Optimal trees were grown to a maximum depth of 4 over both (i) the entire image $(512 \times 512)$ and (ii) the image divided into nonoverlapping $128 \times 128$ image blocks, resulting in full-depth leaf nodes of size $32 \times 32$ for (i) and $8 \times 8$ for (ii). Periodic extension was used at the block boundaries to preserve orthonormality. Scalar quantizers with step sizes that halve with succeedingly deeper tree levels were chosen as members of the admissible set with the root's set being $\{10,40.70,100\}$. First-order entropy was used as the bit rate measure, as entropy coding was assumed to follow the scalar quantization, and an MSE distortion criterion was used. The use of more sophisticated entropy coding methods (e.g., runlength coding) could alter the comparative performance, but not likely substantially.

Fig. 13 shows the operational R-D curves for the "Barbara" image as the quality index $\lambda$ is swept through all positive real numbers, for (a) the optimal wavelet tree, (b) the optimal $512 \times 512$ wavelet packet tree, and (c) the optimal combination of $16128 \times 128$ wavelet packet trees. As can be seen, both (b) and (c) perform substantially better than (a) (about 3-4 dB in PSNR for bit rates of interest or alternatively about $25-30 \%$ reduction in bit rates for desired PSNR's). Also, segmenting the original image into smaller blocks provides better adaptivity and better performance (curve (b) versus curve (c)). Fig. 14 shows a subjective comparison between (a) and (c) for the "Barbara" image at $0.93 \mathrm{bpp}$, with the objective comparisons showing $32.8 \mathrm{~dB}$ and $36.5 \mathrm{~dB}$ respectively. Note the distinct improvement in performance of the optimal WP tree over the optimal wavelet tree in the high frequency stripes of Barbara's trousers, as shown in the blowup.

\section{Optimal Adaptive Wavelet-Packet Split}

It must be mentioned that though we use $128 \times 128$ blocks for the wavelet packet application of Section IV-C, this may not represent the optimal block segmentation for the image, as described in the work on optimal orthonormal tiling of the time-frequency plane in [16], [23]. The optimal split involves 


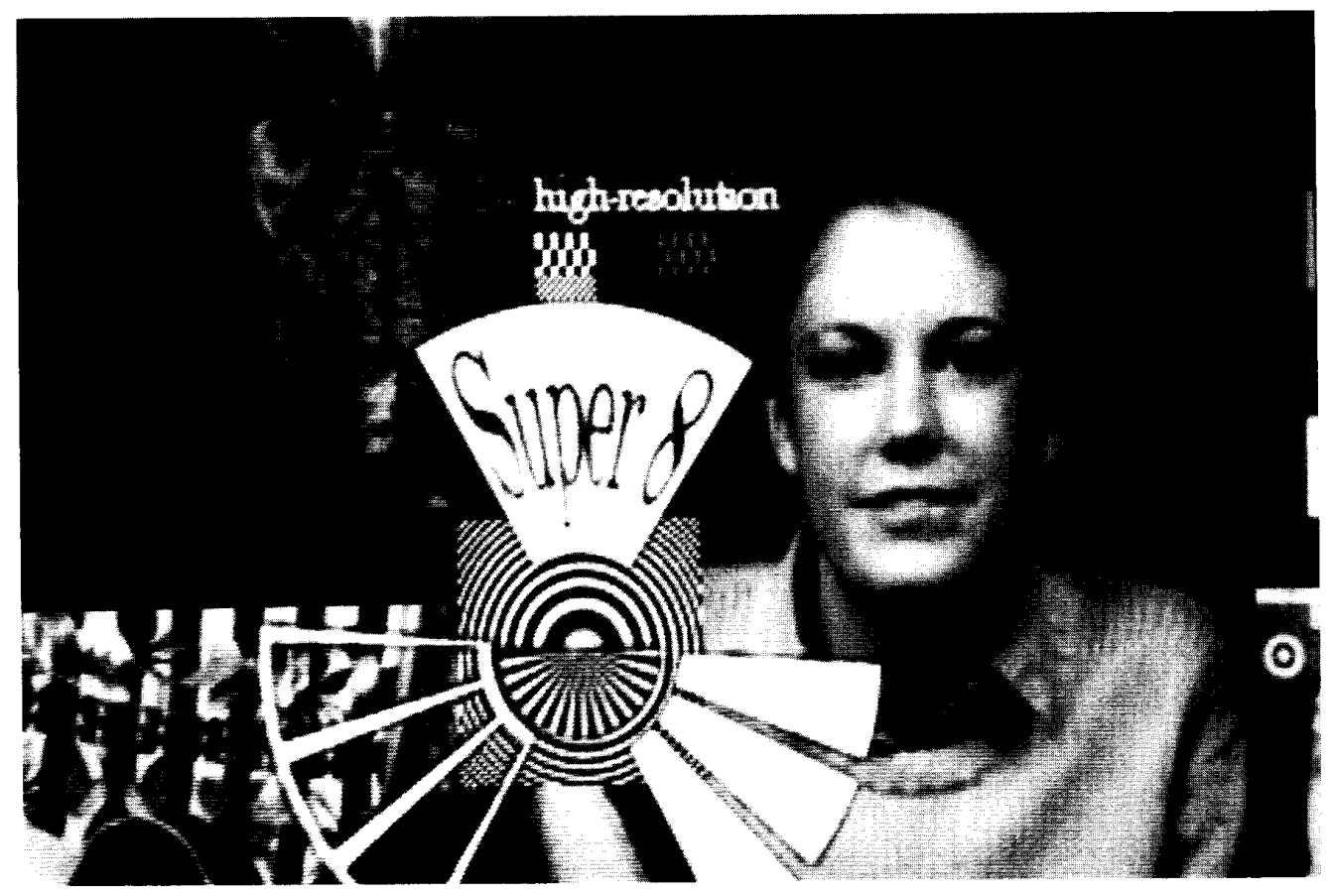

(a)

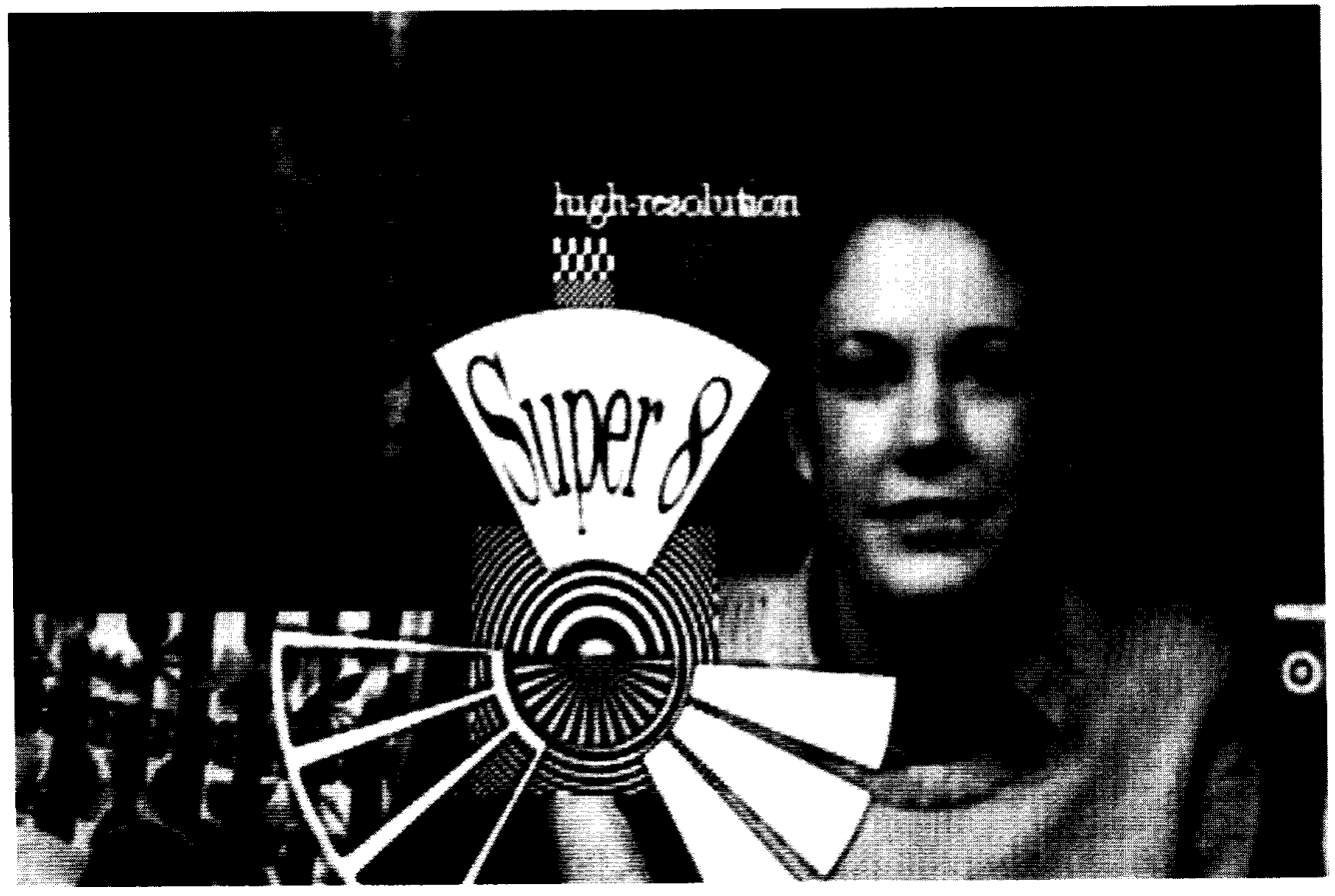

(b)

Fig. 12. Subjective results for (a) JPEG (PSNR $=34 \mathrm{~dB}$ ) and (b) adaptive depth-3 block DCT basis quadtree coding scheme (PSNR $=37 \mathrm{~dB})$ for the "mit" image at a bit rate of 1.37 bits per pixel.

a "double tree" algorithm. In summary, this would involve growing a quadtree segmentation after finding the best wavelet packet basis trees for all desirable block sizes, i.e., a conceptual merging of the applications of Sections VII-B and VII-A (without the DCT basis tree). This would then jointly provide the optimal split as well as the optimal wavelet packet tree for 


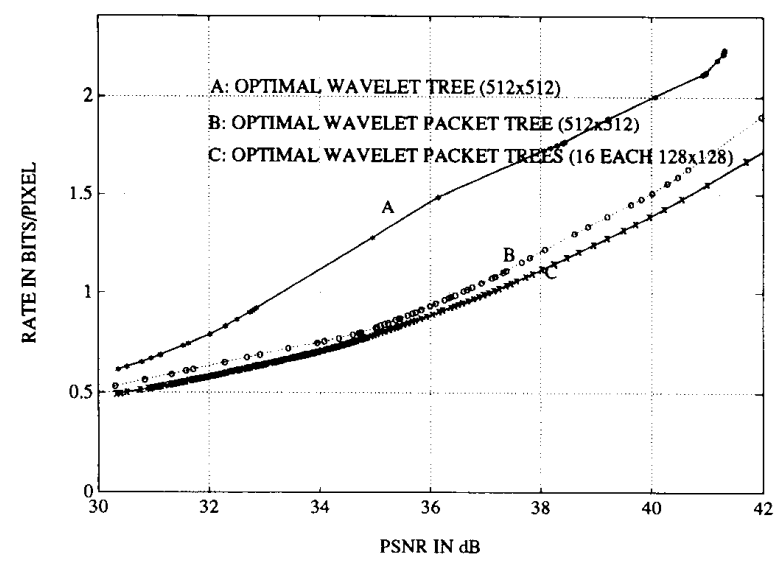

Fig. 13. Comparison of optimal wavelet packet decomposition scheme with optimal wavelet decomposition scheme for the Daubechies D4 set of wavele filters and a $512 \times 512$ portion of the "Barbara" image. Curve (a) is the wavelet case, (b) the signal tree $(512 \times 512)$ wavelet packet case, and (c) the 16 -tree (each $128 \times 128$ ) wavelet packet case. MSE and first-order entropy are used as the distortion and rate measures. The admissible quantizer sets for the WP tree nodes are scalar quantizers which halve in step-size with succeedingly deeper tree levels, with the set at the root being $\{10.40 .70 .100\}$.

each split, as in [16]. As shown in [23], the complexity for the double tree algorithm is $O\left(N(\log N)^{2}\right)$, while that of the wavelet packet algorithm is $O(N(\log N))$.

\section{CONCLUSion}

We have shown a fast $\mathrm{R}-\mathrm{D}$ optimal scheme for coding adaptive trees whose individual nodes spawn off descendents forming a disjoint and complete basis cover for the space spanned by their parent nodes. The scheme presented guarantees operation on the convex hull of the operational $\mathrm{R}-\mathrm{D}$ curve, and uses a fast dynamic programming pruning algorithm to drastically reduce computational complexity. Applications for this coding technique include the CMQW [1] generalized multiresolution wavelet packet decomposition, iterative subband coders, and quadtree structures. Applications to image processing involving wavelet packets as well as DCT quadtrees have been presented with good results.

\section{ACKNOWLEDGMENT}

The authors thank E. Lichtschein for his contribution in developing the wavelet packet algorithm software. They also thank the reviewers, one in particular, for helping make this manuscript more readable.

\section{APPENDIX}

Proof of Lemma 1:

Proof: Denoting $\lambda_{3}=\theta \lambda_{1}+(1-\theta) \lambda_{2}$, where $0 \leq \theta \leq$ 1 , we have

$$
\begin{aligned}
W\left(\lambda_{3}\right) & =W\left(\theta \lambda_{1}+(1-\theta) \lambda_{2}\right) \\
& =\min _{x \in X}\left\{D(x)+\left[\theta \lambda_{1}+(1-\theta) \lambda_{2}\right] R(x)-\lambda_{3} R_{b}\right\}
\end{aligned}
$$

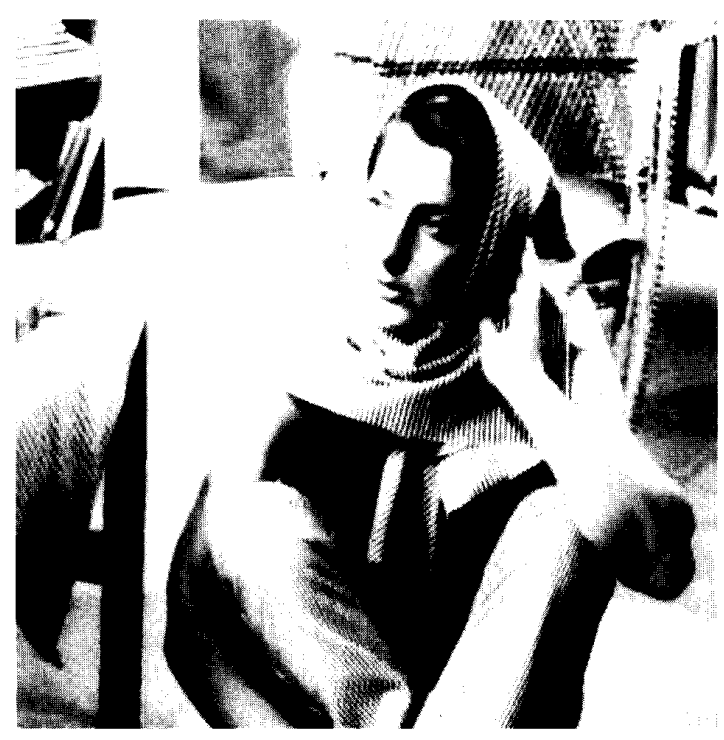

(a)

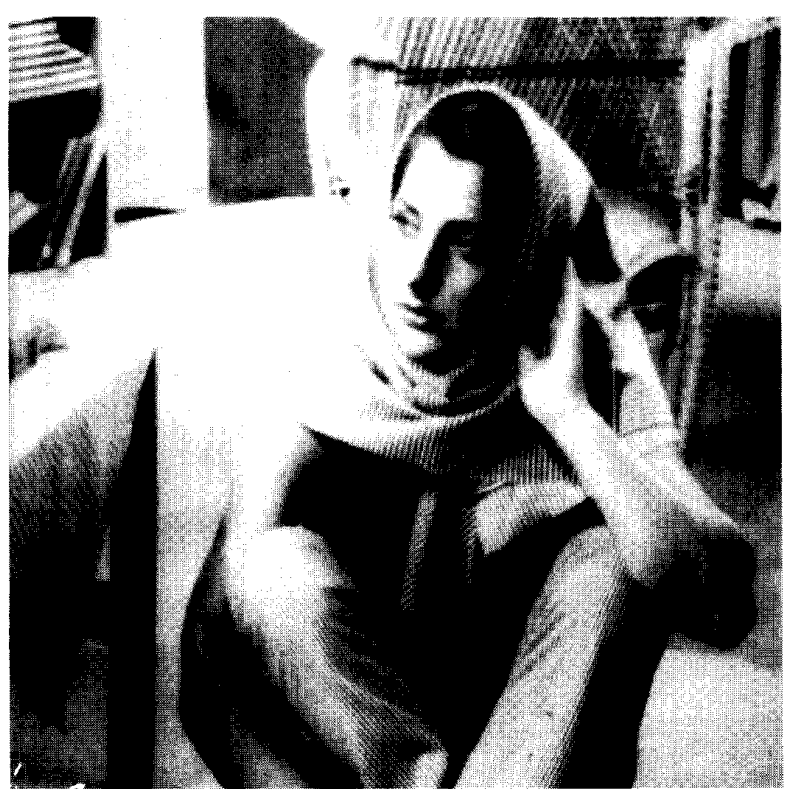

(b)

Fig. 14. Subjective results for the (a) optimal wavelet tree (PSNR = $32.8 \mathrm{~dB}$ ) and (b) the optimal wavelet packet tree for the "Barbara" image $(512 \times 512$ portion) $($ PSNR $=36.4 \mathrm{~dB})$ at a bit rate of 0.92 bits per pixel for the Daubechies D4 set of wavelet filters. (c) a blowup comparing the two schemes for the stripes on Barbara's trousers.

$$
\begin{aligned}
\geq & \min _{x \in X} \theta\left[D(x)+\lambda_{1} R(x)-\lambda_{1} R_{b}\right] \\
& +\min _{x \in X}(1-\theta)\left[D(x)+\lambda_{2} R(x)-\lambda_{2} R_{b}\right] \\
= & \theta W\left(\lambda_{1}\right)+(1-\theta) W\left(\lambda_{2}\right) .
\end{aligned}
$$




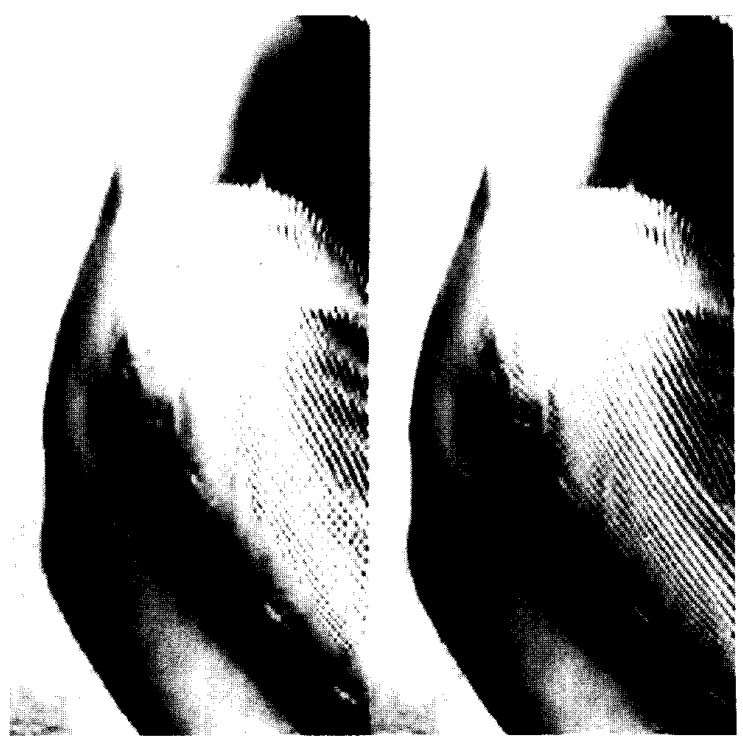

(c)

Fig. 14. (Continued)

Proof of Theorem 2: Denote by $\lambda^{\prime}$ the slope of the convex hull face which "straddles" the budget constraint line on the R-D plane. See Fig. 7. Let us consider the convex-hull face of slope $\lambda^{\prime}$ as a candidate for $\lambda^{*}=\max ^{-1}(W(\lambda))$.

For $\lambda<\lambda^{\prime}$, invoking the "lower rate" operating point on the convex hull of slope $\lambda^{\prime}, \boldsymbol{x}_{\xi}$

$$
\begin{aligned}
W(\lambda)-W\left(\lambda^{\prime}\right)= & \min _{x \in X}\left[D(x)+\lambda R(x)-\lambda R_{b}\right] \\
& -\left[D\left(x_{\xi}\right)+\lambda^{\prime} R\left(x_{\xi}\right)-\lambda^{\prime} R_{b}\right] \\
\leq & {\left[D\left(x_{\xi}\right)+\lambda R\left(x_{\xi}\right)-\lambda R_{b}\right] } \\
& -\left[D\left(x_{\xi}\right)+\lambda^{\prime} R\left(x_{\xi}\right)-\lambda^{\prime} R_{b}\right] \\
\leq & \left(\lambda-\lambda^{\prime}\right)\left(R\left(x_{\xi}\right)-R_{b}\right) \leq 0 .
\end{aligned}
$$

Similarly, for $\lambda>\lambda^{\prime}$, invoking the "higher rate" operating point on the convex hull of slope $\lambda^{\prime}, \boldsymbol{x}_{\gamma}$, we have

$$
W(\lambda)-W\left(\lambda^{\prime}\right) \leq\left(\lambda-\lambda^{\prime}\right)\left(R\left(x_{\gamma}\right)-R_{b}\right) \leq 0 .
$$

Thus for all positive values of $\lambda, W(\lambda) \leq W\left(\lambda^{*}\right)$.

But, by virtue of Lemma 1 , we know that $W(\lambda)$, being concave $\cap$, has a unique maximum value which occurs at a singular slope. (If it were nonsingular, then there exists an $\epsilon>0$, no matter how small, for which $W\left(\lambda^{*}+\epsilon\right)-W\left(\lambda^{*}\right)=$ $\lambda^{*} \epsilon>0$, which contradicts the definition of $\left.W\left(\lambda^{*}\right)\right)$.

Thus $\lambda^{\prime}$ is indeed this unique singular maximum, with the optimal convex hull operating point obviously being $\boldsymbol{x}_{\xi}$.

\section{REFERENCES}

[1] R. Coifman, Y. Meyer, S. Quake, and V. Wickerhauser, "Signal processing and compression with wave packets," Numerical Algorithms Research Group, New Haven, CT: Yale University, 1990.

[2] M.V. Wickerhauser, "Acoustic signal compression with wavelet packets," in Wavelets: A Tutorial in Theory and Applications, C. K. Chui, Ed. New York: Academic, 1992, pp. 679-700.

[3] A. Segall, "Bit allocation and encoding for vector sources," IEEE Trans. Inform. Theory, vol. IT-22, pp. 162-169, Mar. 1976.
[4] Y. Shoham and A. Gersho, "Efficient bit allocation for an arbitrary set of quantizers," IEEE Trans. Acoust., Speech, Signal Processing, vol. 36, pp. $1445-1453$, Sept. 1988.

[5] P. A. Chou, T. Lookabaugh, and R.M. Gray, "Optimal pruning with applications to tree-structured source coding and modeling," IEEE Trans. Inform. Theory, vol. IT-35, pp. 299-315, Mar. 1986

[6] R. M. Gray, Source Coding Theory. Norwell, MA: Kluwer Academic Press, 1990.

[7] A. Gersho and R. M. Gray, Vector Quantization and Signal Compression. Norwell, MA: Kluwer Academic, 1992.

[8] E. A. Riskin, "Optimal bit allocation via the generalized BFOS algorithm," IEEE Trans. Inform. Theory, vol. 37, pp. 299-315, Mar. 1991.

[9] C. T. Chen, "Adaptive transform coding via quadtree-based variable blocksize DCT," in Proc. ICASSP '89, 1989, pp. 1854-1857.

[10] G. J. Sullivan and R. L. Baker, "Efficient quadtree coding of images and video," Proc. ICASSP, vol. 4, pp. 2661-2664, May 1991.

[11] "JPEG technical specification: Revision (DRAFT), joint photographic experts group, ISO/IEC JTC1/SC2/WG8, CCITT SGVIII," Aug. 1990.

[12] S. G. Mallat, "A theory for multiresolution signal decomposition: The wavelet decomposition," IEEE Trans. Patt. Anal. Mach. Intell., vol. 11, pp. $674-693,1989$

[13] M. Vetterli and C. Herley, "Wavelets and filter banks: Theory and design," IEEE Trans. Signal Processing, vol. 40, pp. 2207-2232, Sept. 1992.

[14] H. Malvar, "Efficient signal coding with hierarchical lapped transforms," in Proc. ICASSP '90, 1990, pp. 1519-1522.

[15] S. H. Lee and K.-H. Tzou, "Hierarchical DCT coding of HDTV for ATM networks," in Proc. ICASSP '90, 1990. pp. 2249-2252.

[16] C. Herley, J. Kovacevic, K. Ramchandran, and M. Vetterli, "Arbitrary orthogonal tilings of the time-frequency plane," in IEEE Proc. Int. Symp. on Time-Frequency and Time-Scale Analysis, Oct. 1992, pp. 11-14.

[17] M. Minoux, Mathematical Programming: Theory and Algorithms. New York: Wiley, 1986.

[18] R. Bellman, Dynamic Programming. Princeton, NJ: Princeton Univ. Press, 1957.

[19] W. K. Press, B.P. Flannery, S. A. Teukolsky, and W. T. Vetterling, Numerical Recipes in C: The Art of Scientific Computing. Cambridge, U.K.: Cambridge Univ. Press, 1988.

[20] I. Daubechies, "Orthonormal bases of compactly supported wavelets," Commun. on Pure Appl. Math., vol. XLI, pp. 909996, 1988.

[21] B. Ramamurthi and A. Gersho, "Classified vector quantization of images," IEEE Trans. Commun., vol. 34, pp. 1105-1115, Nov. 1986.

[22] D. J. Vaisey and A. Gersho, "Variable block-size image coding," IEEE Trans. Signal Processing, pp. 2040-2060, Aug. 1987.

[23] C. Herley, J. Kovacevic, K. Ramchandran, and M. Vetterli, "Orthogonal tilings of the time-frequency plane," IEEE Trans. Signal Processing, to be published.

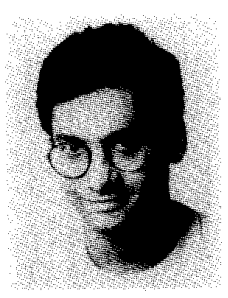

Kannan Ramchandran was born in Madras, India, in 1961. He received his B.S. in E.E. from the City College of New York in 1982 and his M.S. and M. Phil. in E.E. from Columbia University in 1984 and 1992, respectively

From 1982 to 1984, he worked for IBM. In 1984, he joined AT\&T Bell Labs as a Member of the Technical Staff, where he was involved in the design and development of AT\&T's digital loop multiplexers, fast digital loss insertion circuitry for $\mu$-law PCM companders, synchronization and digital scrambling techniques, phase-locked-loops, and custom transmissionIC's in the optical fiber loop carrier systems and the fiber-to-the-home development systems. He is currently on educational leave from AT\&T to finish his Ph.D. dissertation at the Center for Telecommunications Research at Columbia University His research interests include multiresolution source and channel coding, fast algorithms and architectures for image and video coding, multidimensional signal processing and wavelets, and digital communications. 


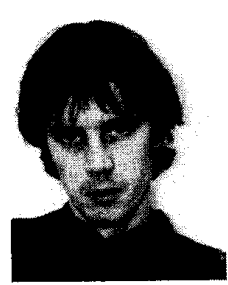

Martin Vetterli (S'86-M'86-SM'90) was born in Switzerland in 1957 . He received the Dipl. El.Ing. degree from the Eidgenössische Technische Hochschule Zürich, Switzerland, in 1981, the Master of Science degree from Stanford University, Stanford, CA, in 1982, and the Doctorat ès Science degree from the Ecole Polytechnique Fédérale de Lausanne, Switzerland, in 1986

In 1982, he was a Research Assistant at Stanford University, and from 1983 to 1986 he was a researcher at the Ecole Polytechnique. He has worked for Siemens and AT\&T Bell Laboratories. In 1986, he joined Columbia University in New York where he is currently Associate Professor of Electrical Engineering, a member of the Center for Telecommunications Research and codirector of the Image and Advanced Television Laboratory. His research interests include wavelets, multirate signal processing, computational complexity, signal processing for telecommunications and digital video processing. He has also worked with the editorial boards of Signal Processing, Image Communication, Annals of Telecommunications and Applied and Computational Harmonic Analysis.

Dr. Vetterli is a member of SIAM and ACM, and a member of the MDSP committee of the IEEE Signal Processing Society. He received the Best Paper Award of EURASIP in 1984 for his paper on multidimensional subband coding, the Research Prize of the Brown Bovery Corporation (Switzerland) in 1986 for his thesis, and the IEEE Signal Processing Society's 1991 Senior Award (DSP Technical Area) for a 1989 Transactions paper with D. LeGall on filter banks for subband coding. 